\title{
REFLECTED BACKWARD STOCHASTIC DIFFERENTIAL EQUATIONS UNDER MONOTONICITY AND GENERAL INCREASING GROWTH CONDITIONS
}

\author{
J.-P. LEPELTIER, ${ }^{* * *}$ \\ A. MATOUSSI ${ }^{* * * *}$ AND \\ M. XU, ${ }^{* * * * *}$ Université du Maine
}

\begin{abstract}
We prove the existence and uniqueness of the solution to certain reflected backward stochastic differential equations (RBSDEs) with one continuous barrier and deterministic terminal time, under monotonicity and general increasing growth conditions on the associated coefficient. As an application, we obtain, in some constraint cases, the price of an American contingent claim as the unique solution of such an RBSDE.
\end{abstract}

Keywords: Reflected backward stochastic differential equation; monotonicity condition; general increasing growth condition; penalization method

2000 Mathematics Subject Classification: Primary 60H10

Secondary 60G40

\section{Introduction}

Nonlinear backward stochastic differential equations (BSDEs) with deterministic terminal time were first introduced by Pardoux and Peng (1990), who proved the existence and uniqueness of adapted solutions with smooth square-integrability assumptions on the associated coefficient $f(t, \omega, y, z)$, and on the terminal condition $\xi$, when the coefficient is Lipschitz in $(y, z)$ uniformly in $(t, \omega)$. Independently, Duffie and Epstein (1992) introduced stochastic differential utilities in economic models as solutions of certain BSDEs. More recently, Briand and Carmona (2000), Pardoux (1999), and Briand et al. (2003) studied the solution of a BSDE with a coefficient $f(t, \omega, y, z)$ that satisfies only monotonicity, continuity, and general increasing growth conditions with respect to $y$, and a Lipschitz condition on $z$. That is, for some real numbers $\mu \in \mathbb{R}$ and $C>0$, the coefficient satisfies

$$
\begin{aligned}
& \left\langle y-y^{\prime}, f(t, y, z)-f\left(t, y^{\prime}, z\right)\right\rangle \\
& \leq \mu\left|y-y^{\prime}\right|^{2} \text { for all }(t, z) \in[0, T] \times \mathbb{R}^{n \times d} \text { and } y, y^{\prime} \in \mathbb{R}^{n} \text {, almost surely (a.s.), } \\
& \left|f(t, y, z)-f\left(t, y, z^{\prime}\right)\right| \\
& \leq C\left|z-z^{\prime}\right| \text { for all }(t, y) \in[0, T] \times \mathbb{R}^{n} \text { and } z, z^{\prime} \in \mathbb{R}^{n \times d} \text {, a.s.; }
\end{aligned}
$$

Received 23 June 2004; revision received 15 October 2004.

* Postal address: Département de Mathématiques, Laboratoire de Statistique et Processus, Université du Maine, 72085

Le Mans cedex 9, France.

** Email address: lepeltier@univ-lemans.fr

*** Email address: anis.matoussi@univ-lemans.fr

**** Email address: mingyu.xu@univ-lemans.fr 
and, for some continuous, increasing function $\varphi: \mathbb{R}_{+} \rightarrow \mathbb{R}_{+}$, it satisfies

$$
|f(t, y, 0)| \leq|f(t, 0,0)|+\varphi(|y|) \quad \text { for all }(t, y) \in[0, T] \times \mathbb{R}^{n} \text {, a.s. }
$$

where $T$ is a fixed, strictly positive terminal time. In Briand and Carmona (2000), the authors considered the case where $\varphi$ is polynomially increasing in $y$.

El Karoui et al. (1997a) introduced the notion of a reflected BSDE on one lower barrier in one dimension: the solution is constrained to remain above a continuous lower-boundary process. More precisely, a solution for such an equation associated with a coefficient $f(t, \omega, y, z)$, a terminal value $\xi$, and a continuous barrier $\left(L_{t}\right)_{0 \leq t \leq T}$ is a triple $\left(Y_{t}, Z_{t}, K_{t}\right)_{0 \leq t \leq T}$ of adapted processes valued in $\mathbb{R}^{1+d+1}$, which satisfies a square-integrability condition,

$$
Y_{t}=\xi+\int_{t}^{T} f\left(s, Y_{s}, Z_{s}\right) \mathrm{d} s+K_{T}-K_{t}-\int_{t}^{T} Z_{s} \mathrm{~d} B_{s}, \quad 0 \leq t \leq T, \text { a.s. }
$$

and $Y_{t} \geq L_{t}, 0 \leq t \leq T$, a.s. (In this equation and all the following, we suppress the explicit dependence of $f$ on $\omega$.) Furthermore, the process $\left(K_{t}\right)_{0 \leq t \leq T}$ is nondecreasing, continuous, and the role of $K_{t}$ is to push the state process upward with minimal energy, in order to keep it above $L$; in this sense, it satisfies $\int_{0}^{T}\left(Y_{S}-L_{S}\right) \mathrm{d} K_{S}=0$. El Karoui et al. (1997a) proved the existence and uniqueness of the solution when $f(t, y, z)$ is Lipschitz in $(y, z)$ uniformly in $(t, \omega)$. Then Matoussi (1997) considered RBSDEs where the coefficient $f$ is continuous and has at most linear growth in $y$ and $z$. In this case, he proved the existence of maximal solutions of the RBSDEs.

In this work, we prove the existence and uniqueness of the solution to a certain type of RBSDE, as Pardoux (1999) did for BSDEs. We work under the assumptions (1.1)-(1.3) on the coefficient $f(t, y, z)$, smooth square-integrability of the barrier $L$, and with the terminal condition $\xi$. In this case, $f$ increases in $y$ at a rate controlled by the positive function $\varphi$, so the techniques of Matoussi (1997) are not adequate.

Our paper is organized as follows. After the presentation of our notation and assumptions in Subsection 2.1, we prove the solution's uniqueness in Subsection 2.2. Then, in Subsection 2.3, we prove its existence in four steps. In the first step, using the penalization method, we show existence under the boundedness condition on the parameters. In step 2, the boundedness condition on the barrier is relaxed. This proves to be our main difficulty, and the solution is not trivial. Then, in the last two steps, we relax the boundedness condition on the coefficient and the terminal condition. In Section 3, we apply this result to characterize, under our working assumptions, the price of an American contingent claim as the unique solution of such an RBSDE. Finally, in Appendix A, several comparison theorems with respect to BSDEs and RBSDEs, which are intensively used in the proof of existence, are presented.

\section{RBSDEs on a fixed finite time interval}

\subsection{Hypotheses and notation}

Let $(\Omega, \mathcal{F}, \mathrm{P})$ be a complete probability space and let $\left(B_{t}\right)_{0 \leq t \leq T}=\left(B_{t}^{1}, B_{t}^{2}, \ldots, B_{t}^{d}\right)_{0 \leq t \leq T}^{\top}$ be a $d$-dimensional Brownian motion defined on a finite interval [0,T], $0<T<\infty$, where ' $\mathrm{T}$, denotes the transpose operator. Denote by $\left\{\mathcal{F}_{t}, 0 \leq t \leq T\right\}$ the natural filtration generated by the Brownian motion $B$ :

$$
\mathcal{F}_{t}=\sigma\left\{B_{s}, 0 \leq s \leq t\right\},
$$

where $\mathcal{F}_{0}$ contains all P-null sets of $\mathcal{F}$. 
We will need the following spaces:

$\mathbf{L}^{2}\left(\mathcal{F}_{t}\right)=\left\{\eta\right.$ : an $\mathcal{F}_{t}$-measurable, random, real-valued variable such that (s.t.)

$$
\left.\mathrm{E}\left[|\eta|^{2}\right]<\infty\right\},
$$

$\mathbf{H}_{n}^{2}(0, T)=\left\{\left(\psi_{t}\right)_{0 \leq t \leq T}:\right.$ a predictable process, valued in $\mathbb{R}^{n}$, s.t. $\left.\mathrm{E} \int_{0}^{T}|\psi(t)|^{2} \mathrm{~d} t<\infty\right\}$,

$\mathbf{S}^{2}(0, T)=\left\{\left(\psi_{t}\right)_{0 \leq t \leq T}:\right.$ a progressively measurable real-valued process s.t.

$$
\left.\mathrm{E}\left[\sup _{0 \leq t \leq T}|\psi(t)|^{2}\right]<\infty\right\}
$$

$\mathbf{A}^{2}(0, T)=\left\{\left(K_{t}\right)_{0 \leq t \leq T}:\right.$ an adapted, continuous, increasing process s.t.

$$
\left.K(0)=0, \mathrm{E}\left[K(T)^{2}\right]<\infty\right\} .
$$

In addition, we will work under the following assumptions.

Assumption 2.1. We assume that $\xi \in \mathbf{L}^{2}\left(\mathcal{F}_{T}\right)$.

Assumption 2.2. There exists a coefficient $f: \Omega \times[0, T] \times \mathbb{R} \times \mathbb{R}^{d} \rightarrow \mathbb{R}$, which is such that, for some continuous, increasing function $\varphi: \mathbb{R}_{+} \rightarrow \mathbb{R}_{+}$and real numbers $\mu$ and $C>0$,

(i) $f(\cdot, y, z)$ is progressively measurable for all $(y, z) \in \mathbb{R} \times \mathbb{R}^{d}$;

(ii) $|f(t, y, 0)| \leq|f(t, 0,0)|+\varphi(|y|)$ for all $(t, y) \in[0, T] \times \mathbb{R}$, a.s.;

(iii) $\mathrm{E} \int_{0}^{T}|f(t, 0,0)|^{2} \mathrm{~d} t<\infty$;

(iv) $\left|f(t, y, z)-f\left(t, y, z^{\prime}\right)\right| \leq C\left|z-z^{\prime}\right|$ for all $(t, y) \in[0, T] \times \mathbb{R}$ and $z, z^{\prime} \in \mathbb{R}^{d}$, a.s.;

(v) $\left(y-y^{\prime}\right)\left(f(t, y, z)-f\left(t, y^{\prime}, z\right)\right) \leq \mu\left(y-y^{\prime}\right)^{2}$ for all $(t, z) \in[0, T] \times \mathbb{R}^{d}$ and $y, y^{\prime} \in \mathbb{R}$, a.s.;

(vi) $y \rightarrow f(t, y, z)$ is continuous for all $(t, z) \in[0, T] \times \mathbb{R}^{d}$, a.s.

Assumption 2.3. There exists a barrier $\left(L_{t}\right)_{0 \leq t \leq T}$ that is a continuous, progressively measurable, real-valued process satisfying $\mathrm{E}\left[\varphi^{2}\left(\sup _{0 \leq t \leq T}\left(\mathrm{e}^{\mu t} L_{t}^{+}\right)\right)\right]<\infty$, with $\left(L_{t}^{+}\right)_{0 \leq t \leq T} \in$ $\mathbf{S}^{2}(0, T)$ and $L_{T} \leq \xi$, a.s.

Now we introduce the definition of the solution of the RBSDE with parameters satisfying Assumptions 2.1-2.3, which is the same as in El Karoui et al. (1997a).

Definition 2.1. We say that $\left(Y_{t}, Z_{t}, K_{t}\right)_{0 \leq t \leq T}$ is a solution of the reflected backward stochastic differential equation with one continuous, reflecting lower barrier $L(\cdot)$, terminal condition $\xi$, and coefficient $f$, if the following conditions hold:

1. $\left(Y_{t}\right)_{0 \leq t \leq T} \in \mathbf{S}^{2}(0, T),\left(Z_{t}\right)_{0 \leq t \leq T} \in \mathbf{H}_{d}^{2}(0, T)$, and $\left(K_{t}\right)_{0 \leq t \leq T} \in \mathbf{A}^{2}(0, T)$;

2. $Y_{t}=\xi+\int_{t}^{T} f\left(s, Y_{s}, Z_{s}\right) \mathrm{d} s+K_{T}-K_{t}-\int_{t}^{T} Z_{s} \mathrm{~d} B_{s}, 0 \leq t \leq T$, a.s.;

3. $Y_{t} \geq L_{t}, 0 \leq t \leq T$;

4. $\int_{0}^{T}\left(Y_{s}-L_{s}\right) \mathrm{d} K_{s}=0$ a.s.

We denote this solution $\operatorname{RBSDE}(\xi, f, L))$. 


\subsection{Uniqueness of the solution of the RBSDE}

We first study the uniqueness of the solution of the $\operatorname{RBSDE}(\xi, f, L)$, under Assumptions 2.1 to 2.3 .

Theorem 2.1. Under Assumptions 2.1-2.3, the $\operatorname{RBSDE}(\xi, f, L)$ has at most one solution $\left(Y_{t}, Z_{t}, K_{t}\right)_{0 \leq t \leq T}$.

Proof. Suppose that

$$
\left(Y_{t}, Z_{t}, K_{t}\right)_{0 \leq t \leq T} \quad \text { and } \quad\left(Y_{t}^{\prime}, Z_{t}^{\prime}, K_{t}^{\prime}\right)_{0 \leq t \leq T}
$$

are two solutions of the $\operatorname{RBSDE}(\xi, f, L)$. Set $\Delta Y=Y-Y^{\prime}, \Delta Z=Z-Z^{\prime}$, and $\Delta K=K-K^{\prime}$. Applying the Itô formula to $\Delta Y^{2}$ on the interval $[t, T]$, and taking expectations on both sides, it follows that

$$
\begin{aligned}
\mathrm{E}\left[\left|\Delta Y_{t}\right|^{2}\right] & +\mathrm{E} \int_{t}^{T}\left|\Delta Z_{s}\right|^{2} \mathrm{~d} s \\
& =2 \mathrm{E} \int_{t}^{T} \Delta Y_{s}\left(f\left(s, Y_{s}, Z_{s}\right)-f\left(s, Y_{s}^{\prime}, Z_{s}^{\prime}\right)\right) \mathrm{d} s+2 \mathrm{E} \int_{t}^{T} \Delta Y_{s} \mathrm{~d}\left(\Delta K_{s}\right) \\
& \leq 2 C \mathrm{E} \int_{t}^{T} \Delta Y_{s} \Delta Z_{s} \mathrm{~d} s+2 \mu \mathrm{E} \int_{t}^{T} \Delta Y_{s}^{2} \mathrm{~d} s \\
& \leq\left(2 C^{2}+\mu\right) \mathrm{E} \int_{t}^{T} \Delta Y_{s}^{2} \mathrm{~d} s+\frac{1}{2} \mathrm{E} \int_{t}^{T}\left|\Delta Z_{s}\right|^{2} \mathrm{~d} s .
\end{aligned}
$$

Here we have used the monotonicity assumption on $y$, the Lipschitz assumption on $z$, and that

$$
\begin{aligned}
\int_{t}^{T} \Delta Y_{s} \mathrm{~d}\left(\Delta K_{s}\right)= & \int_{t}^{T}\left(Y_{s}-L_{s}\right) \mathrm{d} K_{s}+\int_{t}^{T}\left(Y_{s}^{\prime}-L_{s}\right) \mathrm{d} K_{s}^{\prime} \\
& -\int_{t}^{T}\left(Y_{s}-L_{s}\right) \mathrm{d} K_{s}^{\prime}-\int_{t}^{T}\left(Y_{s}^{\prime}-L_{s}\right) \mathrm{d} K_{S} \\
\leq & 0 .
\end{aligned}
$$

We find that

$$
\mathrm{E}\left[\left|\Delta Y_{t}\right|^{2}\right] \leq\left(2 C^{2}+\mu\right) \mathrm{E} \int_{t}^{T} \Delta Y_{s}^{2} \mathrm{~d} s .
$$

From Gronwall's inequality, it follows that $\mathrm{E}\left[\left|\Delta Y_{t}\right|^{2}\right]=\mathrm{E}\left[\left|Y_{t}-Y_{t}^{\prime}\right|^{2}\right]=0,0 \leq t \leq T$, i.e. $Y_{t}=Y_{t}^{\prime}$ a.s. Then, we also have $\mathrm{E} \int_{0}^{T}\left|\Delta Z_{s}\right|^{2} \mathrm{~d} s=\mathrm{E} \int_{0}^{T}\left|Z_{s}-Z_{s}^{\prime}\right|^{2} \mathrm{~d} s=0$, and $K_{t}=K_{t}^{\prime}$ follows.

\subsection{Existence of the solution of the RBSDE}

We will prove the existence of a solution in several steps, as we will see in the following theorem. Comparing with the Lipschitz and the monotonic, linearly increasing cases (see El Karoui et al. (1997a) and Matoussi (1997), respectively), new techniques are needed.

2.3.1. The main result. First, we note that $\left(Y_{t}, Z_{t}, K_{t}\right)_{0 \leq t \leq T}$ solves the $\operatorname{RBSDE}(\xi, f, L)$ if and only if

$$
\left(\bar{Y}_{t}, \bar{Z}_{t}, \bar{K}_{t}\right):=\left(\mathrm{e}^{\lambda t} Y_{t}, \mathrm{e}^{\lambda t} Z_{t}, \int_{0}^{t} \mathrm{e}^{\lambda s} \mathrm{~d} K_{s}\right)
$$


solves the $\operatorname{RBSDE}(\bar{\xi}, \bar{f}, \bar{L})$, where

$$
\begin{aligned}
\bar{\xi} & =\xi \mathrm{e}^{\lambda T}, \\
\bar{f}(t, y, z) & =\mathrm{e}^{\lambda t} f\left(t, \mathrm{e}^{-\lambda t} y, \mathrm{e}^{-\lambda t} z\right)-\lambda y, \\
\bar{L}_{t} & =\mathrm{e}^{\lambda t} L_{t} .
\end{aligned}
$$

If we choose $\lambda=\mu$ then the coefficient $\bar{f}$ satisfies the analogue of Assumption 2.2, but with part (v) replaced by

$$
\left(\mathrm{v}^{\prime}\right)\left(y-y^{\prime}\right)\left(f(t, y, z)-f\left(t, y^{\prime}, z\right)\right) \leq 0 .
$$

Since we are in the one-dimensional case, $\left(\mathrm{v}^{\prime}\right)$ means that $f$ is decreasing in $y$. In addition, the barrier $\bar{L}$ satisfies the following alternative assumption.

Assumption 2.3'.

$$
\begin{aligned}
\mathrm{E}\left[\sup _{0 \leq t \leq T}\left(\bar{L}_{t}^{+}\right)\right] & <\infty, \\
\mathrm{E}\left[\varphi^{2}\left(\sup _{0 \leq t \leq T}\left(\bar{L}_{t}^{+}\right)\right)\right] & =\mathrm{E}\left[\varphi^{2}\left(\sup _{0 \leq t \leq T}\left(\mathrm{e}^{\mu t} L_{t}^{+}\right)\right)\right]<\infty,
\end{aligned}
$$

where $\bar{L}_{t}^{+}$denotes the positive part of $\bar{L}_{t}$.

In the following, we will work with Assumption 2.2', which is Assumption 2.2 with (v) replaced by $\left(\mathrm{v}^{\prime}\right)$, and Assumption 2.3' instead of Assumption 2.3. We first present the following existence theorem, which holds when $f$ does not depend on $z$. It will be proved later.

Theorem 2.2. For any process $\left(V_{t}\right)_{0 \leq t \leq T} \in \mathbf{H}_{d}^{2}(0, T)$, suppose that $f$ satisfies Assumption $2.2^{\prime}$, and that $\left(L_{t}\right)_{0 \leq t \leq T}$ satisfies Assumption 2.3'. Then, there exists a triple $\left(Y_{t}, Z_{t}, K_{t}\right)_{0 \leq t \leq T}$ that satisfies parts 1,3 , and 4 of Definition 2.1, and

$$
Y_{t}=\xi+\int_{t}^{T} f\left(s, Y_{s}, V_{s}\right) \mathrm{d} s+K_{T}-K_{t}-\int_{t}^{T} Z_{s} \mathrm{~d} B_{s}, \quad 0 \leq t \leq T .
$$

With this result, we present the existence of a solution as follows.

Theorem 2.3. Suppose that Assumptions 2.1-2.3 hold. Then, there exists a $\left(Y_{t}, Z_{t}, K_{t}\right)_{0 \leq t \leq T}$ that solves the $\operatorname{RBSDE}(\xi, f, L)$.

Proof. After the transformation of $\left(Y_{t}, Z_{t}, K_{t}\right)_{0 \leq t \leq T}$ in expression (2.1), we consider the $\operatorname{RBSDE}(\xi, f, L)$, whose parameters we take to satisfy Assumptions 2.1, 2.2', and 2.3'. Owing to Theorem 2.2, we can construct a mapping $\Phi$ from the space 8 , defined as the space of progressively measurable, $\left(\mathbb{R} \times \mathbb{R}^{d}\right)$-valued processes $\left(Y_{t}, Z_{t}\right)_{0 \leq t \leq T}$ normed by

$$
\|(Y, Z)\|_{\gamma}:=\left(\mathrm{E} \int_{0}^{T} \mathrm{e}^{\gamma t}\left(\left|Y_{t}\right|^{2}+\left|Z_{t}\right|^{2}\right) \mathrm{d} t\right)^{1 / 2}
$$

(for an appropriate $\gamma \in(0, \infty)$, which will be determined later), into itself.

Given $(U, V) \in \&,(Y, Z)=\Phi(U, V)$ is the unique solution of following RBSDE:

$$
Y_{t}=\xi+\int_{t}^{T} f\left(s, Y_{s}, V_{s}\right) \mathrm{d} s+K_{T}-K_{t}-\int_{t}^{T} Z_{s} \mathrm{~d} B_{s},
$$


i.e. if we define the process

$$
K_{t}=Y_{t}-Y_{0}-\int_{0}^{t} f\left(s, Y_{s}, V_{s}\right) \mathrm{d} s+\int_{0}^{t} Z_{s} \mathrm{~d} B_{s}, \quad 0 \leq t \leq T,
$$

then $(Y, Z, K)$ satisfies parts (1)-(4) of Definition 2.1, with $f(s, y, z)=f\left(s, y, V_{s}\right)$.

Consider another element $\left(u^{\prime}, v^{\prime}\right)$ of $\&$, define $\left(Y^{\prime}, Z^{\prime}\right)=\Phi\left(U^{\prime}, V^{\prime}\right)$, and set

$$
\Delta U=U-U^{\prime}, \quad \Delta V=V-V^{\prime}, \quad \Delta Y=Y-Y^{\prime}, \quad \Delta Z=Z-Z^{\prime} .
$$

Then, by applying the Itô formula to $\mathrm{e}^{\gamma t}\left|\Delta Y_{t}\right|^{2}$ on the interval $[t, T]$, we find that

$$
\begin{gathered}
\mathrm{e}^{\gamma t} \mathrm{E}\left[\left|\Delta Y_{t}\right|^{2}\right]+\mathrm{E} \int_{t}^{T} \mathrm{e}^{\gamma s}\left(\gamma\left|\Delta Y_{s}\right|^{2}+\left|\Delta Z_{s}\right|^{2}\right) \mathrm{d} s \\
=2 \mathrm{E} \int_{t}^{T} \mathrm{e}^{\gamma s} \Delta Y_{s}\left(f\left(s, Y_{s}, V_{s}\right)-f\left(s, Y_{s}^{\prime}, V_{s}^{\prime}\right)\right) \mathrm{d} s+2 \mathrm{E} \int_{t}^{T} \mathrm{e}^{\gamma s} \Delta Y_{s} \mathrm{~d}\left(\Delta K_{s}\right) \\
\leq 2 C^{2} \mathrm{E} \int_{t}^{T} \mathrm{e}^{\gamma s}\left|\Delta Y_{s}\right|^{2} \mathrm{~d} s+\frac{1}{2} \mathrm{E} \int_{t}^{T} \mathrm{e}^{\gamma s}\left|\Delta V_{s}\right|^{2} \mathrm{~d} s,
\end{gathered}
$$

since

$$
\begin{aligned}
\int_{t}^{T} \mathrm{e}^{\gamma s} \Delta Y_{s} \mathrm{~d}\left(\Delta K_{s}\right)= & \int_{t}^{T} \mathrm{e}^{\gamma s}\left(Y_{s}-L_{s}\right) \mathrm{d} K_{s}+\int_{t}^{T} \mathrm{e}^{\gamma s}\left(Y_{s}^{\prime}-L_{s}\right) \mathrm{d} K_{s}^{\prime} \\
& -\int_{t}^{T} \mathrm{e}^{\gamma s}\left(Y_{s}-L_{s}\right) \mathrm{d} K_{s}^{\prime}-\int_{t}^{T} \mathrm{e}^{\gamma s}\left(Y_{s}^{\prime}-L_{s}\right) \mathrm{d} K_{s} \\
\leq & 0 .
\end{aligned}
$$

Hence, if we choose $\gamma=1+2 C^{2}$, it follows that

$$
\begin{aligned}
\int_{t}^{T} \mathrm{e}^{\gamma s}\left(\left|\Delta Y_{s}\right|^{2}+\left|\Delta Z_{s}\right|^{2}\right) \mathrm{d} s & \leq \frac{1}{2} \mathrm{E} \int_{t}^{T} \mathrm{e}^{\gamma s}\left|\Delta V_{s}\right|^{2} \mathrm{~d} s \\
& \leq \frac{1}{2} \mathrm{E} \int_{t}^{T} \mathrm{e}^{\gamma s}\left(\left|\Delta U_{s}\right|^{2}+\left|\Delta V_{s}\right|^{2}\right) \mathrm{d} s
\end{aligned}
$$

Consequently, $\Phi$ is a strict contraction on $\&$ with the norm (2.2), and has a fixed point, which is the unique solution of the $\operatorname{RBSDE}(\xi, f, L)$.

2.3.2. Proof of Theorem 2.2. Let us recall the assumptions on the coefficient $f$ (writing $f(s, y)$ for $\left.f\left(s, y, V_{s}\right)\right)$.

\section{Assumption 2.2".}

(ii') $|f(s, y)| \leq|f(s, 0,0)|+C\left|V_{s}\right|+\varphi(|y|) ;$

(iii') $\mathrm{E} \int_{0}^{T}|f(t, 0)|^{2} \mathrm{~d} t<\infty$;

$\left(\mathrm{v}^{\prime \prime}\right)\left(y-y^{\prime}\right)\left(f(s, y)-f\left(s, y^{\prime}\right)\right) \leq 0$;

(vi') $y \mapsto f(s, y)$ is continuous for all $s \in[0, T]$, a.s.

We point out that we always denote by $c>0$ a constant whose value can change from line to line. 
The proof of Theorem 2.2 will be done in four steps, as follows.

1. Using a penalization method, we prove existence under the assumption that

$$
|\xi|^{2}+\sup _{0 \leq t \leq T}|f(t, 0)|^{2}+\sup _{0 \leq t \leq T} L_{t}^{+} \leq c .
$$

2. Approximating the barrier $L$, we prove existence under Assumption $2.3^{\prime}$ and the boundedness assumption on $\xi$ and $f(t, 0)$, i.e.

$$
|\xi|^{2}+\sup _{0 \leq t \leq T}|f(t, 0)|^{2} \leq c .
$$

3. By approximation, we prove the existence of the solution under the assumptions that $\xi \geq c$ and $\inf _{0 \leq t \leq T} f(t, 0) \geq c$.

4. Finally, and again by approximation, we prove the existence of the solution under the assumptions that $\xi \in \mathbf{L}^{2}\left(\mathcal{F}_{T}\right)$ and $f(t, 0) \in \mathbf{H}_{1}^{2}(0, T)$.

Step 1. We shall need the following lemma, in view of the estimation.

Lemma 2.1. Suppose that $f$ satisfies Assumption 2.2" and that (2.3) holds. Then, there exists a triple $\left(Y_{t}^{*}, Z_{t}^{*}, K_{t}^{*}\right)_{0 \leq t \leq T}$ that satisfies

$$
Y_{t}^{*}=\xi+\int_{t}^{T} f\left(s, Y_{s}^{*}\right) \mathrm{d} s+K_{T}^{*}-K_{t}^{*}-\int_{t}^{T} Z_{s}^{*} \mathrm{~d} B_{s} ;
$$

$Y_{t}^{*} \geq L_{t}, 0 \leq t \leq T$, a.s.; $\sup _{0 \leq t \leq T}\left|Y_{t}^{*}\right| \leq c$; and $Z^{*} \in \mathbf{H}_{d}^{2}(0, T)$, with $K^{*}$ increasing, $K_{0}^{*}=0$, and $K_{T}^{*} \leq c$.

Proof. Consider the random variable $\bar{\xi}:=\max \left\{\sup _{0 \leq t \leq T} L_{t}^{+}, \xi\right\} \geq 0$. By (2.3), it follows that $|\bar{\xi}| \leq c$. Set $\bar{L}_{t}=\mathrm{E}\left[\bar{\xi} \mid \mathcal{F}_{t}\right]$. The process $\bar{L}_{t}$ is a bounded martingale and, by the Itô representation theorem, there exists a process $\bar{Z} \in \mathbf{H}_{d}^{2}(0, T)$ such that

$$
\begin{aligned}
\bar{L}_{t} & =\bar{L}_{0}+\int_{0}^{t} \bar{Z}_{s} \mathrm{~d} B_{s}=\bar{\xi}-\int_{t}^{T} \bar{Z}_{s} \mathrm{~d} B_{s} \\
& =\bar{\xi}+\int_{t}^{T} f\left(s, \bar{L}_{s}\right) \mathrm{d} s-\int_{t}^{T} f\left(s, \bar{L}_{s}\right) \mathrm{d} s-\int_{t}^{T} \bar{Z}_{s} \mathrm{~d} B_{s} .
\end{aligned}
$$

The process $K_{t}^{*}=\int_{0}^{t} f^{-}\left(s, \bar{L}_{s}\right) \mathrm{d} s+(\bar{\xi}-\xi) \mathbf{1}_{\{t=T\}}$ is uniformly bounded and increasing, since

$$
K_{T}^{*} \leq T\left(\sup _{0 \leq t \leq T} f(t, 0)+\varphi\left(\sup _{0 \leq t \leq T} \bar{L}_{t}\right)\right)+|\bar{\xi}|+|\xi| \leq c .
$$

Now consider $(\tilde{Y}, \tilde{Z})$, the solution of the following BSDE, where $\tilde{f}(t, y)=f\left(t, y-K_{t}^{*}\right)$ :

$$
\tilde{Y}_{t}=\xi+K_{T}^{*}+\int_{t}^{T} \tilde{f}\left(s, \tilde{Y}_{s}\right) \mathrm{d} s-\int_{t}^{T} \tilde{Z}_{s} \mathrm{~d} B_{s}
$$

Since

$$
\left|\xi+K_{T}^{*}\right|+\sup _{0 \leq t \leq T}|\tilde{f}(t, 0)| \leq|\xi|+K_{T}^{*}+\sup _{0 \leq t \leq T}|f(t, 0)|+\varphi\left(\left|K_{T}^{*}\right|\right) \leq c
$$


from the proof of the first step of Proposition 2.4 of Pardoux (1999), the BSDE (2.7) has a unique solution $\left(\tilde{Y}_{t}, \tilde{Z}_{t}\right)_{0 \leq t \leq T}$ and $\tilde{Y}$ is uniformly bounded. Now, if we set $Y_{t}^{*}=\tilde{Y}_{t}-K_{t}^{*}$ and $Z_{t}^{*}=\tilde{Z}_{t}$, it is easy to check that $\left(Y^{*}, Z^{*}\right)$ satisfies

$$
Y_{t}^{*}=\xi+\int_{t}^{T} f\left(s, Y_{s}^{*}\right) \mathrm{d} s+K_{T}^{*}-K_{t}^{*}-\int_{t}^{T} Z_{s}^{*} \mathrm{~d} B_{s},
$$

with

$$
\sup _{0 \leq t \leq T}\left|Y_{t}^{*}\right| \leq \sup _{0 \leq t \leq T}\left|\tilde{Y}_{t}\right|+K_{T}^{*} \leq c .
$$

On the other hand, (2.6) can be rewritten as

$$
\bar{L}_{t}+K_{t}^{*}=\xi+K_{T}^{*}+\int_{t}^{T} \tilde{f}\left(s, \bar{L}_{s}+K_{s}^{*}\right) \mathrm{d} s-\int_{t}^{T} f^{+}\left(s, \bar{L}_{s}\right) \mathrm{d} s-\int_{t}^{T} \bar{Z}_{s} \mathrm{~d} B_{s} .
$$

Since $\int_{0}^{t} f^{+}\left(s, \bar{L}_{s}\right) \mathrm{d} s$ is an increasing process, by the generalized comparison theorem (Theorem A.1 in Appendix A) we have $Y_{t}^{*}+K_{t}^{*} \geq \bar{L}_{t}+K_{t}^{*}, 0 \leq t \leq T$, and so

$$
Y_{t}^{*} \geq \bar{L}_{t} \geq L_{t}, \quad 0 \leq t \leq T .
$$

We can now start to prove the existence of the solution under the assumption (2.3). Consider the penalized BSDEs

$$
Y_{t}^{n}=\xi+\int_{t}^{T} f\left(s, Y_{s}^{n}\right) \mathrm{d} s+n \int_{t}^{T}\left(Y_{s}^{n}-L_{s}\right)^{-} \mathrm{d} s-\int_{t}^{T} Z_{s}^{n} \mathrm{~d} B_{s}, \quad n \in \mathbb{N} .
$$

By setting $f_{n}(s, y)=f(s, y)+n\left(y-L_{s}\right)^{-}$, it is easy to check that $f_{n}$ satisfies the assumptions of Proposition 2.4 in Pardoux (1999), so it follows that each of these penalized BSDEs admits a unique solution $\left(Y_{t}^{n}, Z_{t}^{n}\right)_{0 \leq t \leq T}$.

Write

$$
K_{t}^{n}=n \int_{0}^{t}\left(Y_{s}^{n}-L_{s}\right)^{-} \mathrm{d} s .
$$

Let us now prove the a-priori estimate of $\left(Y_{t}^{n}, Z_{t}^{n}, K_{t}^{n}\right)_{0 \leq t \leq T}$, uniformly in $n$. To do so, we consider the BSDE

$$
\tilde{Y}_{t}=\xi+\int_{t}^{T} f\left(s, \tilde{Y}_{s}\right) \mathrm{d} s-\int_{t}^{T} \tilde{Z}_{s} \mathrm{~d} B_{s}
$$

with coefficient $f(t, \cdot)$. By the result of the first step of Proposition 2.4 of Pardoux (1999), $\sup _{0 \leq t \leq T}\left|\tilde{Y}_{t}\right| \leq c$. Obviously, for $(s, y) \in[0, T] \times \mathbb{R}, f_{n}(s, y) \geq f(s, y)$; therefore, by Theorem 2.4 of Pardoux (1999) we obtain

$$
Y_{t}^{n} \geq \tilde{Y}_{t}, \quad 0 \leq t \leq T, \quad \text { a.s. }
$$

On the other hand, by Lemma 2.1 there exists a triple $\left(Y_{t}^{*}, Z_{t}^{*}, K_{t}^{*}\right)_{0 \leq t \leq T}$ that satisfies (2.5) with $Y_{t}^{*} \geq L_{t}, 0 \leq t \leq T$, and $\sup _{0 \leq t \leq T}\left|Y_{t}^{*}\right| \leq c$. Moreover, the triple $\left(Y^{*}, Z^{*}, K^{*}\right)$ satisfies

$$
\begin{aligned}
Y_{t}^{*} & =\xi+\int_{t}^{T} f\left(s, Y_{s}^{*}\right) \mathrm{d} s+n \int_{t}^{T}\left(Y_{s}^{*}-L_{s}\right)^{-} \mathrm{d} s+K_{T}^{*}-K_{t}^{*}-\int_{t}^{T} Z_{s}^{*} \mathrm{~d} B_{s} \\
& =\xi+\int_{t}^{T} f_{n}\left(s, Y_{s}^{*}\right) \mathrm{d} s+K_{T}^{*}-K_{t}^{*}-\int_{t}^{T} Z_{s}^{*} \mathrm{~d} B_{s} .
\end{aligned}
$$


Using Theorem A.1, we find that $Y_{t}^{*} \geq Y_{t}^{n}, 0 \leq t \leq T$. Then, using (2.8),

$$
Y_{t}^{*} \geq Y_{t}^{n} \geq \tilde{Y}_{t}, \quad 0 \leq t \leq T,
$$

follows and, since $Y^{*}$ and $\tilde{Y}$ are uniformly bounded in the interval $[0, T]$,

$$
\sup _{0 \leq t \leq T}\left|Y_{t}^{n}\right| \leq \max \left\{\sup _{0 \leq t \leq T}\left|Y_{t}^{*}\right|, \sup _{0 \leq t \leq T}\left|\tilde{Y}_{t}\right|\right\} \leq c,
$$

where $c$ is a constant independent of $n$. Furthermore, for each $n \in \mathbb{N}$,

$$
\left|f\left(s, Y_{s}^{n}\right)\right| \leq|f(t, 0)|+\varphi\left(\sup _{0 \leq t \leq T}\left|Y_{s}^{n}\right|\right) \leq c .
$$

Now we apply the Itô formula to $\left|Y_{t}^{n}\right|^{2}$ on the interval $[t, T]$ and take expectations, to find that

$$
\begin{aligned}
\mathrm{E}\left[\left|Y_{t}^{n}\right|^{2}\right]+\mathrm{E} \int_{t}^{T}\left|Z_{s}^{n}\right|^{2} \mathrm{~d} s \leq & \mathrm{E}\left[|\xi|^{2}\right]+\mathrm{E} \int_{t}^{T}\left|Y_{s}^{n}\right|^{2} \mathrm{~d} s+\mathrm{E} \int_{t}^{T}|f(s, 0)|^{2} \mathrm{~d} s \\
& +\alpha \mathrm{E}\left[\sup _{0 \leq t \leq T}\left(L_{t}^{+}\right)^{2}\right]+\frac{1}{\alpha} \mathrm{E}\left[\left(K_{T}^{n}-K_{t}^{n}\right)^{2}\right],
\end{aligned}
$$

where $\alpha$ is a positive number. We rewrite the $\operatorname{BSDE}\left(\xi, f_{n}, L\right)$ as

$$
K_{T}^{n}-K_{t}^{n}=Y_{t}^{n}-\xi-\int_{t}^{T} f\left(s, Y_{s}^{n}\right) \mathrm{d} s+\int_{t}^{T} Z_{s}^{n} \mathrm{~d} B_{s} .
$$

Hence, by (2.3), (2.9), and (2.10),

$$
\begin{aligned}
\mathrm{E}\left[\left(K_{T}^{n}-K_{t}^{n}\right)^{2}\right] & \leq 2 \mathrm{E}\left[\left|Y_{t}^{n}\right|^{2}\right]+2 \mathrm{E}\left[|\xi|^{2}\right]+2 T \mathrm{E} \int_{t}^{T}\left|f\left(s, Y_{s}^{n}\right)\right|^{2} \mathrm{~d} s+2 \mathrm{E} \int_{t}^{T}\left|Z_{s}^{n}\right|^{2} \mathrm{~d} s \\
& \leq c+2 \mathrm{E} \int_{t}^{T}\left|Z_{s}^{n}\right|^{2} \mathrm{~d} s .
\end{aligned}
$$

Next we substitute (2.13) into (2.11) and set $\alpha=4$; from (2.3) and (2.9), it follows that

$$
\mathrm{E} \int_{0}^{T}\left|Z_{s}^{n}\right|^{2} \mathrm{~d} s \leq c .
$$

Using (2.13) again, we find that

$$
\mathrm{E}\left[\left(K_{T}^{n}\right)^{2}\right] \leq c
$$

Notice that, for all $n \in \mathbb{N}$ and for all $(s, y) \in[0, T] \times \mathbb{R}, f_{n}(s, y) \leq f_{n+1}(s, y)$. Therefore, by Theorem 2.4 of Pardoux (1999), we have $Y_{t}^{n} \leq Y_{t}^{n+1}, 0 \leq t \leq T$, a.s. Hence,

$$
Y_{t}^{n} \nearrow Y_{t}, \quad 0 \leq t \leq T, \quad \text { a.s. }
$$

In view of (2.9), we have

$$
\sup _{0 \leq t \leq T}\left|Y_{t}\right| \leq c
$$

and, by the dominated convergence theorem,

$$
\mathrm{E} \int_{0}^{T}\left(Y_{t}^{n}-Y_{t}\right)^{2} \mathrm{~d} t \rightarrow 0 \quad \text { as } n \rightarrow \infty .
$$


We will now prove that the sequence $Y^{n}$ converges in the space $\mathbf{S}^{2}(0, T)$. Applying the Itô formula to $\left|Y_{t}^{n}-Y_{t}^{p}\right|^{2}$, for $n, p \in \mathbb{N}$, on the interval $[t, T]$, we find that

$$
\begin{aligned}
& \mathrm{E}\left[\left|Y_{t}^{n}-Y_{t}^{p}\right|^{2}\right]+\mathrm{E} \int_{t}^{T}\left|Z_{s}^{n}-Z_{s}^{p}\right|^{2} \mathrm{~d} s \\
& \quad=2 \mathrm{E} \int_{t}^{T}\left(f\left(s, Y_{s}^{n}\right)-f\left(s, Y_{s}^{p}\right)\right)\left(Y_{s}^{n}-Y_{s}^{p}\right) \mathrm{d} s+2 \mathrm{E} \int_{t}^{T}\left(Y_{s}^{n}-Y_{s}^{p}\right) \mathrm{d}\left(K_{s}^{n}-K_{s}^{p}\right) \\
& \quad \leq 2 \mathrm{E} \int_{t}^{T}\left(Y_{s}^{n}-L_{s}\right)^{-} \mathrm{d} K_{s}^{p}+2 \mathrm{E} \int_{t}^{T}\left(Y_{s}^{p}-L_{s}\right)^{-} \mathrm{d} K_{s}^{n} .
\end{aligned}
$$

Let us state the following lemma. Since $Y^{n}$ and $f\left(t, Y^{n}\right)$ are uniformly bounded, its proof is similar to that of Lemma 6.1 in El Karoui et al. (1997a), so we omit it.

Lemma 2.2. For $0 \leq t \leq T$, the limit satisfies $Y_{t} \geq L_{t}$ a.s., and

$$
\mathrm{E}\left[\sup _{0 \leq t \leq T}\left(\left|Y_{t}^{n}-L_{t}\right|^{-}\right)^{2}\right] \rightarrow 0 \quad \text { as } n \rightarrow \infty .
$$

As in El Karoui et al. (1997a), from this lemma and (2.14) we deduce that, for the first term in the last line of (2.16),

$$
\mathrm{E} \int_{t}^{T}\left(Y_{s}^{n}-L_{s}\right)^{-} \mathrm{d} K_{s}^{p} \leq\left(\mathrm{E}\left[\sup _{0 \leq t \leq T}\left(\left|Y_{t}^{n}-L_{t}\right|^{-}\right)^{2}\right]\right)^{1 / 2}\left(\mathrm{E}\left[\left(K_{T}^{p}\right)^{2}\right]\right)^{1 / 2} \rightarrow 0
$$

as $n, p \rightarrow \infty$. Similarly,

$$
\mathrm{E} \int_{t}^{T}\left(Y_{s}^{p}-L_{s}\right)^{-} \mathrm{d} K_{s}^{n} \rightarrow 0 \quad \text { as } n, p \rightarrow \infty
$$

Hence, from (2.16),

$$
\mathrm{E} \int_{0}^{T}\left|Z_{s}^{n}-Z_{s}^{p}\right|^{2} \mathrm{~d} s \rightarrow 0 \quad \text { as } n, p \rightarrow \infty
$$

and there exists a process $Z \in \mathbf{H}_{d}^{2}(0, T)$ such that

$$
\mathrm{E} \int_{0}^{T}\left|Z_{s}^{n}-Z_{s}\right|^{2} \mathrm{~d} s \rightarrow 0 \quad \text { as } n \rightarrow \infty .
$$

Then, by the Burkholder-Davis-Gundy (BDG) inequality, it follows that

$$
\begin{aligned}
\mathrm{E}\left[\sup _{0 \leq t \leq T}\left|Y_{t}^{n}-Y_{t}^{p}\right|^{2}\right] \leq & 4 \mathrm{E} \int_{0}^{T}\left(Y_{s}^{n}-L_{s}\right)^{-} \mathrm{d} K_{s}^{p}+4 \mathrm{E} \int_{0}^{T}\left(Y_{s}^{p}-L_{s}\right)^{-} \mathrm{d} K_{s}^{n} \\
& +c \int_{0}^{T}\left|Z_{s}^{n}-Z_{s}^{p}\right|^{2} \mathrm{~d} s \\
& \rightarrow 0
\end{aligned}
$$

as $n, p \rightarrow \infty$. Finally,

$$
\mathrm{E}\left[\sup _{0 \leq t \leq T}\left|Y_{t}^{n}-Y_{t}\right|^{2}\right] \rightarrow 0 \quad \text { as } n \rightarrow \infty
$$


By (2.15) and the fact that $f(s, y)$ is continuous and decreasing in $y$, we have $f\left(s, Y_{s}^{n}\right) \searrow$ $f\left(s, Y_{s}\right), 0 \leq s \leq T$. Moreover, $\left|f\left(s, Y_{s}^{n}\right)\right| \leq c$ and, using the monotone convergence theorem, we deduce that

$$
\mathrm{E} \int_{0}^{T}\left[f\left(t, Y_{t}^{n}\right)-f\left(t, Y_{t}\right)\right]^{2} \mathrm{~d} t \rightarrow 0 .
$$

Now let us consider the convergence of the sequence $K^{n}$. For $n, p \in \mathbb{N}$, rewrite $K^{n}$ and $K^{p}$ in forward form (i.e. between 0 and $t$ ), as in (2.12), and consider their difference. Using the BDG inequality, we obtain

$$
\begin{aligned}
\mathrm{E}\left[\sup _{0 \leq t \leq T}\left|K_{t}^{n}-K_{t}^{p}\right|^{2}\right] \leq & 2\left|Y_{0}^{n}-Y_{0}^{p}\right|^{2}+2 \mathrm{E}\left[\sup _{0 \leq t \leq T}\left|Y_{t}^{n}-Y_{t}^{p}\right|^{2}\right] \\
& +2 \mathrm{E}\left[\sup _{0 \leq t \leq T}\left(\int_{0}^{t}\left(f\left(s, Y_{s}^{n}\right)-f\left(s, Y_{s}^{p}\right)\right) \mathrm{d} s\right)^{2}\right] \\
& +2 \mathrm{E}\left[\left(\sup _{0 \leq t \leq T}\left|\int_{0}^{t}\left(Z_{s}^{n}-Z_{s}^{p}\right) \mathrm{d} B_{s}\right|\right)^{2}\right] \\
\leq & 2\left|Y_{0}^{n}-Y_{0}^{p}\right|^{2}+2 \mathrm{E}\left[\sup _{0 \leq t \leq T}\left|Y_{t}^{n}-Y_{t}^{p}\right|^{2}\right] \\
& +2 T \mathrm{E} \int_{0}^{T}\left(f\left(s, Y_{s}^{n}\right)-f\left(s, Y_{s}^{p}\right)\right)^{2} \mathrm{~d} s+c \mathrm{E} \int_{0}^{T}\left|Z_{s}^{n}-Z_{s}^{p}\right|^{2} \mathrm{~d} s .
\end{aligned}
$$

By (2.15), (2.17), and (2.18), it follows that

$$
\mathrm{E}\left[\sup _{0 \leq t \leq T}\left|K_{t}^{n}-K_{t}^{p}\right|^{2}\right] \rightarrow 0 \quad \text { as } n, p \rightarrow \infty,
$$

so there exists an increasing process $K$ in $\mathbf{A}^{2}(0, T)$ such that

$$
\mathrm{E}\left[\sup _{0 \leq t \leq T}\left|K_{t}^{n}-K_{t}\right|^{2}\right] \rightarrow 0 \quad \text { as } n \rightarrow \infty
$$

and $\left(Y_{t}, Z_{t}, K_{t}\right)_{0 \leq t \leq T} \in \mathbf{S}^{2}(0, T) \times \mathbf{H}_{d}^{2}(0, T) \times \mathbf{A}^{2}(0, T)$ satisfies property 2 of Definition 2.1 .

From Lemma 2.2 , we know that property 3 of Definition 2.1 is true; it remains to check property 4. Since $\left(Y_{t}^{n}, K_{t}^{n}\right)_{0 \leq t \leq T}$ tends to $\left(Y_{t}, K_{t}\right)_{0 \leq t \leq T}$ uniformly in $t$ in probability, the measure $\mathrm{d} K^{n}$ converges to $\mathrm{d} K$ weakly in probability, so that $\int_{0}^{T}\left(Y_{t}^{n}-L_{t}\right) \mathrm{d} K_{t}^{n} \rightarrow \int_{0}^{T}\left(Y_{t}-L_{t}\right) \mathrm{d} K_{t}$ in probability as $n \rightarrow \infty$. Obviously, $\int_{0}^{T}\left(Y_{t}-L_{t}\right) \mathrm{d} K_{t} \geq 0$, while, on the other hand, for each $n \in \mathbb{N}, \int_{0}^{T}\left(Y_{t}^{n}-L_{t}\right) \mathrm{d} K_{t}^{n} \leq 0$. Hence,

$$
\int_{0}^{T}\left(Y_{t}-L_{t}\right) \mathrm{d} K_{t}=0 \quad \text { a.s. }
$$

Consequently, $(Y, Z, K)$ is a solution of the $\operatorname{RBSDE}(\xi, f, L)$, under the assumption (2.3).

Step 2. Now we consider the case of a barrier $L$ that satisfies Assumption 2.3', that is,

$$
\mathrm{E}\left[\varphi^{2}\left(\sup _{0 \leq t \leq T}\left(L_{t}^{+}\right)\right)\right]<\infty
$$

with $L^{+} \in \mathbf{S}^{2}(0, T)$ and $L_{T} \leq \xi$, even when $\xi$ and $f(t, 0)$ are uniformly bounded. 
Under Assumption 2.2" and (2.4), we know that there exist constants $c_{1}$ and $c_{2}$ such that $\xi \leq c_{1}$ and $f(t, 0) \leq c_{2}$. Set $c^{\prime}=\max \left\{c_{1}, c_{2} T\right\}$. Then $\left(Y_{t}, Z_{t}, K_{t}\right)_{0 \leq t \leq T}$ is the solution of the $\operatorname{RBSDE}(\xi, f, L)$ if and only if $\left(Y_{t}^{\prime}, Z_{t}^{\prime}, K_{t}^{\prime}\right)_{0 \leq t \leq T}$ is the solution of the $\operatorname{RBSDE}\left(\xi^{\prime}, f^{\prime}, L^{\prime}\right)$, where

$$
\left(Y_{t}^{\prime}, Z_{t}^{\prime}, K_{t}^{\prime}\right)=\left(Y_{t}+c_{2} t-2 c^{\prime}, Z_{t}, K_{t}\right)
$$

and

$$
\begin{aligned}
\xi^{\prime} & =\xi+c_{2} T-2 c^{\prime}, \\
f^{\prime}(t, y) & =f\left(t, y-\left(c_{2} t-2 c^{\prime}\right)\right)-c_{2}, \\
L_{t}^{\prime} & =L_{t}+c_{2} t-2 c^{\prime} .
\end{aligned}
$$

Since $c^{\prime} \geq c_{2} T, L^{\prime} \leq L$, which implies that $L^{\prime}$ satisfies Assumption 2.3'. In fact, the triple

$$
\left(Y_{t}^{\prime}, Z_{t}^{\prime}, K_{t}^{\prime}\right)_{0 \leq t \leq T}
$$

satisfies property 2 of Definition 2.1 for the $\operatorname{RBSDE}\left(\xi^{\prime}, f^{\prime}, L^{\prime}\right)$

$$
\begin{aligned}
Y_{t}^{\prime} & =Y_{t}+c_{2} t-2 c^{\prime} \\
& =\xi^{\prime}+\int_{t}^{T} f^{\prime}\left(s, Y_{s}^{\prime}\right) \mathrm{d} s+K_{T}^{\prime}-K_{t}^{\prime}-\int_{t}^{T} Z_{s}^{\prime} \mathrm{d} B_{s},
\end{aligned}
$$

and $Y_{t}^{\prime}=Y_{t}+c_{2} t-2 c^{\prime} \geq L_{t}+c_{2} t-2 c^{\prime}=L_{t}^{\prime}$, with

$$
\int_{0}^{T}\left(Y_{t}^{\prime}-L_{t}^{\prime}\right) \mathrm{d} K_{t}^{\prime}=\int_{0}^{T}\left(Y_{t}-L_{t}\right) \mathrm{d} K_{t}=0 .
$$

Now we consider the $\operatorname{RBSDE}\left(\xi^{\prime}, f^{\prime}, L^{\prime}\right)$. Obviously, (2.4) also holds for $\xi^{\prime}$ and $f^{\prime}$; in fact,

$$
\left|\xi^{\prime}\right|+\sup _{0 \leq t \leq T}\left|f^{\prime}(t, 0)\right| \leq|\xi|+c_{2} T-2 c^{\prime}+\sup _{0 \leq t \leq T}|f(t, 0)|+\varphi\left(2 c^{\prime}\right)+c_{2} \leq c .
$$

It follows directly that $f^{\prime}$ satisfies Assumption $2.2^{\prime \prime}$ with $\varphi^{\prime}(y)=|f(t, 0)|+c_{2}+\varphi\left(|y|+2 c^{\prime}\right)$, which is still a continuous, increasing, positive function. Moreover, since $f$ is decreasing on $y$ and $2 c^{\prime}-c_{2} t \geq 0$, we have

$$
\begin{aligned}
\xi^{\prime} & =\xi+c_{2} T-2 c^{\prime} \leq \xi-c^{\prime} \leq 0, \\
f^{\prime}(t, 0) & =f\left(t, 0-\left(c_{2} t-2 c^{\prime}\right)\right)-c_{2} \leq f(t, 0)-c_{2} \leq 0 .
\end{aligned}
$$

We now need the following lemma.

Lemma 2.3. Assume that $f$ satisfies Assumption 2.2', that (2.4) holds, and that the barrier $L$ satisfies Assumption 2.3'. Furthermore, suppose that

$$
\xi \leq 0 \text { and } f(t, 0) \leq 0 .
$$

Then there exists a triple $\left(Y_{t}, Z_{t}, K_{t}\right)_{0 \leq t \leq T}$ that solves the $R B S D E(\xi, f, L)$.

By this lemma, there exists a unique $\left(Y_{t}^{\prime}, Z_{t}^{\prime}, K_{t}^{\prime}\right)_{0 \leq t \leq T}$ that solves the $\operatorname{RBSDE}\left(\xi^{\prime}, f^{\prime}, L^{\prime}\right)$. Then we know that the $\operatorname{RBSDE}(\xi, f, L)$ has the unique solution $\left(Y_{t}, Z_{t}, K_{t}\right)_{0 \leq t \leq T}$. 
Proof of Lemma 2.3. For $n \in \mathbb{N}$, set $L^{n}=L \wedge n$; then $\sup _{0 \leq t \leq T}\left(L_{t}^{n}\right)^{+} \leq n$. By Step 1, we know that, for all $n$, there exists a triple $\left(Y_{t}^{n}, Z_{t}^{n}, K_{t}^{n}\right)_{0 \leq t \leq T}$ that satisfies

$$
\begin{aligned}
& Y_{t}^{n}=\xi+\int_{t}^{T} f\left(s, Y_{s}^{n}\right) \mathrm{d} s+K_{T}^{n}-K_{t}^{n}-\int_{t}^{T} Z_{s}^{n} \mathrm{~d} B_{s}, \\
& Y_{t}^{n} \geq L_{t}^{n}, \quad 0 \leq t \leq T, \quad \text { and } \quad \int_{0}^{T}\left(Y_{t}^{n}-L_{t}^{n}\right) \mathrm{d} K_{t}^{n}=0 .
\end{aligned}
$$

By Theorem A.1, $Y_{t}^{n} \geq \tilde{Y}_{t}, 0 \leq t \leq T$, where $\left(\tilde{Y}_{t}, \tilde{Z}_{t}\right)_{0 \leq t \leq T} \in \mathbf{S}^{2}(0, T) \times \mathbf{H}_{d}^{2}(0, T)$ is the solution of the classic $\operatorname{BSDE}(\xi, f)$

$$
\tilde{Y}_{t}=\xi+\int_{t}^{T} f\left(s, \tilde{Y}_{s}\right) \mathrm{d} s-\int_{t}^{T} \tilde{Z}_{s} \mathrm{~d} B_{s} .
$$

Let us consider the $\operatorname{RBSDE}\left(\xi^{+}, 0, L^{+}\right)$; by Proposition 2.3 of El Karoui et al. (1997a), the Snell envelope of $L_{t}^{+} \mathbf{1}_{\{t<T\}}+\xi^{+} \mathbf{1}_{\{t=T\}}$ is the solution of this linear RBSDE, so

$$
\begin{aligned}
& \bar{Y}_{t}=\underset{\tau \in \mathcal{T}_{t, T}}{\operatorname{ess} \sup } \mathrm{E}\left[L_{\tau}^{+} \mathbf{1}_{\{\tau<T\}}+\xi^{+} \mathbf{1}_{\{\tau=T\}} \mid \mathscr{F}_{t}\right]=\underset{\tau \in \mathcal{T}_{t, T}}{\operatorname{ess} \sup } \mathrm{E}\left[L_{\tau}^{+} \mid \mathscr{F}_{t}\right] \\
& =\bar{K}_{T}-\bar{K}_{t}-\int_{t}^{T} \bar{Z}_{s} \mathrm{~d} B_{s},
\end{aligned}
$$

in view of the fact that $L_{T}^{+}=\xi^{+}=0$, where $\widetilde{T}_{t, T}$ denotes the set of stopping times $t \leq \tau \leq T$. The processes $\bar{K}$ and $\bar{Z}$ come from the Doob-Meyer decomposition of the Snell envelope and the Itô representation of the martingale part. Since $\bar{Y}_{t} \geq L_{t}^{+} \geq 0$ and $f$ is decreasing, $f\left(t, \bar{Y}_{t}\right) \leq f(t, 0) \leq 0$, which implies that $f^{+}\left(t, \bar{Y}_{t}\right)=0$. So, $\left(\bar{Y}_{t}, \bar{Z}_{t}, \bar{K}_{t}\right)_{0 \leq t \leq T}$ is still the solution of the $\operatorname{RBSDE}\left(\xi^{+}, f^{+}, L^{+}\right)$. Moreover, notice that the Snell envelope is the smallest supermartingale that dominates the process $L^{+}$, and that it is positive. Therefore, we have

$$
\begin{aligned}
\mathrm{E}\left[\sup _{0 \leq t \leq T}\left(\bar{Y}_{t}\right)^{2}\right] & \leq \mathrm{E}\left[\sup _{0 \leq t \leq T}\left(\mathrm{E}\left[\sup _{0 \leq t \leq T} L_{t}^{+} \mid \mathcal{F}_{t}\right]\right)^{2}\right] \\
& \leq \mathrm{E}\left[\sup _{0 \leq t \leq T} \mathrm{E}\left[\left(\sup _{0 \leq t \leq T} L_{t}^{+}\right)^{2} \mid \mathcal{F}_{t}\right]\right] \\
& \leq \mathrm{E}\left[\sup _{0 \leq t \leq T}\left(L_{t}^{+}\right)^{2}\right]
\end{aligned}
$$

so $\left(\bar{Y}_{t}\right)_{0 \leq t \leq T} \in \mathbf{S}^{2}(0, T)$, since $\left(L_{t}^{+}\right)_{0 \leq t \leq T} \in \mathbf{S}^{2}(0, T)$.

Notice that $\xi^{+} \geq \xi, f^{+}(t, y) \geq f(t, y),(t, y) \in[0, T] \times R$, and $L_{t}^{+} \geq L_{t} \geq L_{t}^{n}$, $0 \leq t \leq T$, for $n \in \mathbb{N}$. Therefore, by Theorem A.2, we get $Y_{t}^{n} \leq \bar{Y}_{t}, 0 \leq t \leq T$, and, consequently,

$$
\mathrm{E}\left[\sup _{0 \leq t \leq T}\left(Y_{t}^{n}\right)^{2}\right] \leq \max \left\{\mathrm{E}\left[\sup _{0 \leq t \leq T}\left(\tilde{Y}_{t}\right)^{2}\right], \mathrm{E}\left[\sup _{0 \leq t \leq T}\left(\bar{Y}_{t}\right)^{2}\right]\right\} \leq c .
$$

Since $L_{t}^{n} \leq L_{t}^{n+1}, 0 \leq t \leq T$, from Theorem A.2 we have $Y_{t}^{n} \nearrow Y_{t}, 0 \leq t \leq T$. From (2.21) and Fatou's lemma, we get

$$
\mathrm{E}\left[\sup _{0 \leq t \leq T}\left(Y_{t}\right)^{2}\right] \leq c
$$


and it follows from the dominated convergence theorem that

$$
\mathrm{E} \int_{0}^{T}\left|Y_{t}^{n}-Y_{t}\right|^{2} \mathrm{~d} t \rightarrow 0 \quad \text { as } n \rightarrow \infty .
$$

In order to prove the convergence of $\left(Z^{n}, K^{n}\right)$, we first need a-priori estimates. Applying the Itô formula to $\left|Y_{t}^{n}\right|^{2}$, and using the fact that $a b \leq \alpha a^{2}+(1 / \alpha) b^{2}$ for all $\alpha>0$, we find that

$$
\begin{aligned}
\mathrm{E}\left[\left|Y_{t}^{n}\right|^{2}\right]+\mathrm{E} \int_{t}^{T}\left|Z_{s}^{n}\right|^{2} \mathrm{~d} s \leq & \mathrm{E}\left[|\xi|^{2}\right]+\mathrm{E} \int_{t}^{T}\left|Y_{s}^{n}\right|^{2} \mathrm{~d} s+\mathrm{E} \int_{t}^{T}|f(s, 0)|^{2} \mathrm{~d} s \\
& +\alpha \mathrm{E}\left[\sup _{0 \leq t \leq T}\left|Y_{t}^{n}\right|^{2}\right]+\frac{1}{\alpha} \mathrm{E}\left[\left(K_{T}^{n}-K_{t}^{n}\right)^{2}\right],
\end{aligned}
$$

where $\alpha$ is a positive number. We rewrite the $\operatorname{RBSDE}\left(\xi, f, L^{n}\right)$ in forward form, as in (2.12) in Step 1, then square and take expectations on both sides, from which it follows that

$$
\mathrm{E}\left[\left(K_{T}^{n}-K_{t}^{n}\right)^{2}\right] \leq 2 \mathrm{E}\left[\left|Y_{t}^{n}\right|^{2}\right]+2 \mathrm{E}\left[|\xi|^{2}\right]+2 \mathrm{E}\left[\left(\int_{t}^{T} f\left(s, Y_{s}^{n}\right) \mathrm{d} s\right)^{2}\right]+2 \mathrm{E} \int_{t}^{T}\left|Z_{s}^{n}\right|^{2} \mathrm{~d} s .
$$

Since

$$
\bar{Y}_{t} \geq Y_{t}^{n} \geq \tilde{Y}_{t}, \quad 0 \leq t \leq T,
$$

and from the monotonicity property of $f(t, y)$, it follows that

$$
f\left(t, \bar{Y}_{t}\right) \leq f\left(t, Y_{t}^{n}\right) \leq f\left(t, \tilde{Y}_{t}\right) .
$$

Then, from (2.20),

$$
\mathrm{E}\left[\left(\int_{0}^{T} f\left(t, \tilde{Y}_{t}\right) \mathrm{d} t\right)^{2}\right] \leq 2 \mathrm{E}\left[|\xi|^{2}\right]+2\left(\tilde{Y}_{0}\right)^{2}+2 \mathrm{E} \int_{t}^{T}\left|\tilde{Z}_{s}\right|^{2} \mathrm{~d} s \leq c .
$$

On the other hand, due to the fact that $\bar{Y}$ is the Snell envelope of $L^{+}, \sup _{0 \leq t \leq T} \bar{Y}_{t} \geq$ $\sup _{0 \leq t \leq T} L_{t}^{+}$. Then $\bar{Y}_{t} \leq \bar{L}_{t}$, since the process $\bar{L}_{t}=\mathrm{E}\left[\sup _{0 \leq t \leq T} L_{t}^{+} \mid \mathcal{F}_{t}\right]$ is a martingale that dominates $L^{+}$. Notice that

$$
\begin{aligned}
\mathrm{E}\left[\sup _{0 \leq t \leq T} \bar{Y}_{t}\right] & \leq \mathrm{E}\left[\sup _{0 \leq t \leq T} \bar{L}_{t}\right]=\mathrm{E}\left[\sup _{0 \leq t \leq T} \mathrm{E}\left[\sup _{0 \leq t \leq T} L_{t}^{+} \mid \mathcal{F}_{t}\right]\right] \\
& \leq \mathrm{E}\left[\mathrm{E}\left[\sup _{0 \leq t \leq T} L_{t}^{+} \mid \mathcal{F}_{t}\right]\right] \\
& =\mathrm{E}\left[\sup _{0 \leq t \leq T} L_{t}^{+}\right]:
\end{aligned}
$$

it follows that $\sup _{0 \leq t \leq T} \bar{Y}_{t}=\sup _{0 \leq t \leq T} L_{t}^{+}$. Then, from Assumption 2.3', it further follows that

$$
\begin{aligned}
\mathrm{E}\left[\left(\int_{0}^{T} f\left(t, \bar{Y}_{t}\right) \mathrm{d} t\right)^{2}\right] & \leq \mathrm{E}\left[\int_{0}^{T}\left(2 f^{2}(t, 0)+2 \varphi^{2}\left(\sup _{0 \leq t \leq T} L_{t}^{+}\right)\right) \mathrm{d} t\right] \\
& \leq c+2 T \mathrm{E}\left[\varphi^{2}\left(\sup _{0 \leq t \leq T} L_{t}^{+}\right)\right] \\
& \leq c
\end{aligned}
$$


and so we have

$$
\mathrm{E}\left[\left(\int_{0}^{T} f\left(t, Y_{t}^{n}\right) \mathrm{d} t\right)^{2}\right] \leq \max \left\{\mathrm{E}\left[\left(\int_{0}^{T} f\left(t, \bar{Y}_{t}\right) \mathrm{d} t\right)^{2}\right], \mathrm{E}\left[\left(\int_{0}^{T} f\left(t, \tilde{Y}_{t}\right) \mathrm{d} t\right)^{2}\right]\right\} \leq c
$$

and, from (2.24),

$$
\mathrm{E}\left[\left(K_{T}^{n}-K_{t}^{n}\right)^{2}\right] \leq c+2 \mathrm{E} \int_{t}^{T}\left|Z_{s}^{n}\right|^{2} \mathrm{~d} s .
$$

If we substitute (2.26) into (2.23), set $\alpha=4$, and use (2.4) and (2.21), it then follows that

$$
\mathrm{E} \int_{0}^{T}\left|Z_{s}^{n}\right|^{2} \mathrm{~d} s \leq c .
$$

Using (2.26) again, we get

$$
\mathrm{E}\left[\left(K_{T}^{n}\right)^{2}\right] \leq c .
$$

Now if $n, p \in \mathbb{N}, n \geq p$, then $L_{t}^{n} \geq L_{t}^{p}, 0 \leq t \leq T$. By applying the Itô formula to $\left|Y_{t}^{n}-Y_{t}^{p}\right|^{2}$, and recalling that $f$ satisfies Assumption $2.3^{\prime}\left(\mathrm{v}^{\prime \prime}\right)$ we find that

$$
\begin{aligned}
\mathrm{E}\left[\mid Y_{t}^{n}-\right. & \left.\left.Y_{t}^{p}\right|^{2}\right]+\mathrm{E} \int_{t}^{T}\left|Z_{s}^{n}-Z_{s}^{p}\right|^{2} \mathrm{~d} s \\
= & 2 \mathrm{E} \int_{t}^{T}\left[f\left(s, Y_{s}^{n}\right)-f\left(s, Y_{s}^{p}\right)\right]\left(Y_{s}^{n}-Y_{s}^{p}\right) \mathrm{d} s+2 \mathrm{E} \int_{t}^{T}\left(Y_{s}^{n}-Y_{s}^{p}\right) \mathrm{d}\left(K_{s}^{n}-K_{s}^{p}\right) \\
\leq & 2 \mathrm{E} \int_{t}^{T}\left(Y_{s}^{n}-L_{s}^{n}\right) \mathrm{d} K_{s}^{n}+2 \mathrm{E} \int_{t}^{T}\left(Y_{s}^{p}-L_{s}^{p}\right) \mathrm{d} K_{s}^{p}-2 \mathrm{E} \int_{t}^{T}\left(Y_{s}^{n}-L_{s}^{n}\right) \mathrm{d} K_{s}^{p} \\
& -2 \mathrm{E} \int_{t}^{T}\left(Y_{s}^{p}-L_{s}^{p}\right) \mathrm{d} K_{s}^{n}+2 \mathrm{E} \int_{t}^{T}\left(L_{s}^{n}-L_{s}^{p}\right) \mathrm{d}\left(K_{s}^{n}-K_{s}^{p}\right) \\
\leq & 2 \mathrm{E} \int_{t}^{T}\left(L_{s}^{n}-L_{s}^{p}\right) \mathrm{d} K_{s}^{n}-2 \mathrm{E} \int_{t}^{T}\left(L_{s}^{n}-L_{s}^{p}\right) \mathrm{d} K_{s}^{p} \\
\leq & 2 \mathrm{E} \int_{t}^{T}\left(L_{s}^{n}-L_{s}^{p}\right) \mathrm{d} K_{s}^{n} .
\end{aligned}
$$

Since $L_{t}-L_{t}^{n} \downarrow 0$ for each $t \in[0, T]$, and $L_{t}-L_{t}^{n}$ is continuous, by the Dini theorem the convergence holds uniformly on the interval $[0, T]$, i.e.

$$
\mathrm{E}\left[\sup _{0 \leq t \leq T}\left(L_{t}-L_{t}^{n}\right)^{2}\right] \rightarrow 0 \quad \text { as } n \rightarrow \infty .
$$

Then, using (2.27),

$$
\begin{aligned}
\mathrm{E} \int_{0}^{T}\left|Z_{s}^{n}-Z_{s}^{p}\right|^{2} \mathrm{~d} s & \leq 2 \mathrm{E}\left[\sup _{0 \leq t \leq T}\left(L_{s}^{n}-L_{s}^{p}\right) K_{T}^{n}\right] \\
& \leq 2\left(\mathrm{E}\left[\sup _{0 \leq t \leq T}\left(L_{s}^{n}-L_{s}^{p}\right)^{2}\right]\right)^{1 / 2}\left(\mathrm{E}\left[\left(K_{T}^{n}\right)^{2}\right]\right)^{1 / 2} \\
& \leq c\left(\mathrm{E}\left[\sup _{0 \leq t \leq T}\left(L_{s}^{n}-L_{s}^{p}\right)^{2}\right]\right)^{1 / 2} \\
& \rightarrow 0
\end{aligned}
$$


as $n, p \rightarrow \infty$, so there exists a process $\left(Z_{t}\right)_{0 \leq t \leq T} \in \mathbf{H}_{d}^{2}(0, T)$ such that, as $n \rightarrow \infty$,

$$
\mathrm{E} \int_{0}^{T}\left|Z_{s}^{n}-Z_{s}\right|^{2} \mathrm{~d} s \rightarrow 0 .
$$

Furthermore, by the Itô formula,

$$
\begin{aligned}
\sup _{0 \leq t \leq T}\left|Y_{t}^{n}-Y_{t}^{p}\right|^{2} \leq & 2 \sup _{0 \leq t \leq T} \int_{t}^{T}\left(L_{s}^{n}-L_{s}^{p}\right) \mathrm{d}\left(K_{s}^{n}-K_{s}^{p}\right) \\
& +2 \sup _{0 \leq t \leq T}\left|\int_{t}^{T}\left(Y_{s}^{n}-Y_{s}^{p}\right)\left(Z_{s}^{n}-Z_{s}^{p}\right) \mathrm{d} B_{s}\right| .
\end{aligned}
$$

Taking the expectation of both sides, by the BDG inequality and (2.27) we find that

$$
\begin{aligned}
\mathrm{E}\left[\sup _{0 \leq t \leq T}\left|Y_{t}^{n}-Y_{t}^{p}\right|^{2}\right] \leq & 2\left(\mathrm{E}\left[\sup _{0 \leq t \leq T}\left(L_{s}^{n}-L_{s}^{p}\right)^{2}\right]\right)^{1 / 2}\left(\mathrm{E}\left[\left(K_{T}^{n}\right)^{2}\right]\right)^{1 / 2} \\
& +c \mathrm{E}\left[\sup _{0 \leq t \leq T}\left|Y_{s}^{n}-Y_{s}^{p}\right|^{2} \int_{0}^{T}\left|Z_{s}^{n}-Z_{s}^{p}\right|^{2} \mathrm{~d} s\right] \\
\leq & c\left(\mathrm{E}\left[\sup _{0 \leq t \leq T}\left(L_{s}^{n}-L_{s}^{p}\right)^{2}\right]\right)^{1 / 2}+\frac{1}{2} \mathrm{E}\left[\sup _{0 \leq t \leq T}\left|Y_{s}^{n}-Y_{s}^{p}\right|^{2}\right] \\
& +c \mathrm{E} \int_{0}^{T}\left|Z_{s}^{n}-Z_{s}^{p}\right|^{2} \mathrm{~d} s .
\end{aligned}
$$

Hence, by (2.22), (2.29), and (2.28), as $n, p \rightarrow \infty$,

$$
\mathrm{E}\left[\sup _{0 \leq t \leq T}\left|Y_{t}^{n}-Y_{t}^{p}\right|^{2}\right] \rightarrow 0
$$

which implies that there exists a process $\left(Y_{t}\right)_{0 \leq t \leq T} \in \mathbf{S}^{2}(0, T)$ such that, as $n \rightarrow \infty$,

$$
\mathrm{E}\left[\sup _{0 \leq t \leq T}\left|Y_{t}^{n}-Y_{t}\right|\right] \rightarrow 0
$$

Moreover, since $f$ is continuous and decreasing on $y$, with $Y_{t}^{n} \nearrow Y_{t}, 0 \leq t \leq T$, we also have

$$
f\left(t, Y_{t}^{n}\right)-f\left(t, Y_{t}\right) \searrow 0, \quad 0 \leq t \leq T
$$

By the monotone limit theorem, we find that $\int_{0}^{T}\left[f\left(t, Y_{t}^{n}\right)-f\left(t, Y_{t}\right)\right] \mathrm{d} t \searrow 0$ while, from (2.25) and the convergence of $Y_{t}^{n}$, we have $\bar{Y}_{t} \geq Y_{t} \geq \tilde{Y}_{t}, 0 \leq t \leq T$; given the monotonic condition on $f$, it follows that

$$
\mathrm{E}\left[\left(\int_{0}^{T} f\left(t, Y_{t}\right) \mathrm{d} t\right)^{2}\right] \leq \max \left\{\mathrm{E}\left[\left(\int_{0}^{T} f\left(t, \bar{Y}_{t}\right) \mathrm{d} t\right)^{2}\right], \mathrm{E}\left[\left(\int_{0}^{T} f\left(t, \tilde{Y}_{t}\right) \mathrm{d} t\right)^{2}\right]\right\} \leq c
$$

with $\mathrm{E}\left[\left(\int_{0}^{T} f\left(t, Y_{t}^{n}\right) \mathrm{d} t\right)^{2}\right] \leq c$. We then deduce that

$$
\mathrm{E}\left[\int_{0}^{T}\left(f_{n}\left(t, Y_{t}^{n}\right)-f\left(t, Y_{t}\right)\right) \mathrm{d} t\right]^{2} \rightarrow 0 \quad \text { as } n \rightarrow \infty .
$$


Since $\mathrm{E}\left[\left(K_{T}^{n}\right)^{2}\right] \leq c$, it follows that $\mathrm{E}\left[\left(K_{t}^{n}\right)^{2}\right] \leq c$ for each $t \in[0, T]$. Also, the sequence $\left(K_{t}^{n}\right)$ has a weak limit $K_{t}$ in $\mathbf{L}^{2}\left(\mathcal{F}_{t}\right)$, with $\mathrm{E}\left[\left(K_{t}\right)^{2}\right] \leq c$. Therefore, for $0 \leq t \leq T,\left(Y_{t}, Z_{t}, K_{t}\right)_{0 \leq t \leq T}$ satisfies

$$
Y_{t}=\xi+\int_{t}^{T} f\left(s, Y_{s}\right) \mathrm{d} s+K_{T}-K_{t}-\int_{t}^{T} Z_{s} \mathrm{~d} B_{s}
$$

We must prove the convergence of $\left\{K^{n}\right\}$ in a stronger sense. For this, we rewrite (2.19) and (2.32) in the forward form with respect to $K$ (as in (2.12)), and then consider their difference. It follows that

$$
\begin{aligned}
\sup _{0 \leq t \leq T}\left|K_{t}^{n}-K_{t}\right|^{2} \leq & 2\left|Y_{0}^{n}-Y_{0}\right|^{2}+2 \sup _{0 \leq t \leq T}\left|Y_{t}^{n}-Y_{t}\right|^{2} \\
& +2 \sup _{0 \leq t \leq T}\left|\int_{0}^{t}\left(f\left(s, Y_{s}^{n}\right)-f\left(s, Y_{s}\right)\right) \mathrm{d} s\right|^{2} \\
& +2 \sup _{0 \leq t \leq T}\left|\int_{0}^{t}\left(Z_{s}^{n}-Z_{s}\right) \mathrm{d} B_{s}\right| .
\end{aligned}
$$

Taking expectations on both sides, and using the BDG inequality and the fact that $f\left(s, Y_{s}^{n}\right) \geq$ $f\left(s, Y_{s}\right)$, it follows in turn that

$$
\begin{aligned}
\mathrm{E}\left[\sup _{0 \leq t \leq T}\left|K_{t}^{n}-K_{t}\right|^{2}\right] \leq & 2\left|Y_{0}^{n}-Y_{0}\right|^{2}+2 \mathrm{E}\left[\sup _{0 \leq t \leq T}\left|Y_{t}^{n}-Y_{t}\right|^{2}\right] \\
& +2 \mathrm{E}\left[\left(\int_{0}^{T}\left[f\left(s, Y_{s}^{n}\right)-f\left(s, Y_{s}\right)\right] \mathrm{d} s\right)^{2}\right] \\
& +c \mathrm{E} \int_{0}^{T}\left|Z_{s}^{n}-Z_{s}\right|^{2} \mathrm{~d} s .
\end{aligned}
$$

Then, by (2.30), (2.31), and (2.29), we deduce that, as $n \rightarrow \infty$,

$$
\mathrm{E}\left[\sup _{0 \leq t \leq T}\left|K_{t}^{n}-K_{t}\right|^{2}\right] \rightarrow 0 .
$$

The last thing to check is that $(Y, Z, K)$ also satisfies properties 3 and 4 of Definition 2.1 . Since, for each $n \in \mathbb{N}$ and $0 \leq t \leq T, Y_{t}^{n} \geq L_{t}^{n}$ almost surely, with $Y_{t}^{n} \nearrow Y_{t}$ and $L_{t}^{n} \nearrow L_{t}$, we have that $Y_{t} \geq L_{t}$ almost surely. We know that the processes $K^{n}$ are increasing, so the limit $K$ is also increasing. Notice that $\left(Y_{t}^{n}, K_{t}^{n}\right)_{0 \leq t \leq T}$ tends to $\left(Y_{t}, K_{t}\right)_{0 \leq t \leq T}$ uniformly in $t$ in probability, so the measure $\mathrm{d} K^{n}$ converges to $\mathrm{d} K$ weakly in probability, and $\left(L_{t}^{n}\right)_{0 \leq t \leq T}$ converges to $\left(L_{t}\right)_{0 \leq t \leq T}$ in $\mathbf{S}^{2}(0, T)$ as $n \rightarrow \infty$. Hence,

$$
\begin{aligned}
& \mathrm{E} \int_{0}^{T}\left(Y_{t}-L_{t}\right) \mathrm{d} K_{t}-\mathrm{E} \int_{0}^{T}\left(Y_{t}^{n}-L_{t}^{n}\right) \mathrm{d} K_{t}^{n} \\
&= \mathrm{E} \int_{0}^{T}\left(Y_{t}-Y_{t}^{n}\right) \mathrm{d} K_{t}^{n}+\mathrm{E} \int_{0}^{T}\left(Y_{t}-L_{t}\right) \mathrm{d}\left(K_{t}-K_{t}^{n}\right)+\mathrm{E} \int_{0}^{T}\left(L_{t}^{n}-L_{t}\right) \mathrm{d} K_{t}^{n} \\
& \leq\left(\mathrm{E}\left[\sup _{0 \leq t \leq T}\left(Y_{t}^{n}-Y_{t}\right)^{2}\right]\right)^{1 / 2}\left(\mathrm{E}\left[\left(K_{T}^{n}\right)^{2}\right]\right)^{1 / 2}+\mathrm{E} \int_{0}^{T}\left(Y_{t}-L_{t}\right) \mathrm{d}\left(K_{t}^{n}-K_{t}\right) \\
&+\left(\mathrm{E}\left[\sup _{0 \leq t \leq T}\left(L_{t}-L_{t}^{n}\right)^{2}\right]\right)^{1 / 2}\left(\mathrm{E}\left[\left(K_{T}^{n}\right)^{2}\right]\right)^{1 / 2} \\
& \rightarrow 0
\end{aligned}
$$


If $\mathrm{E} \int_{0}^{T}\left(Y_{t}^{n}-L_{t}^{n}\right) \mathrm{d} K_{t}^{n}=0$ then $\mathrm{E} \int_{0}^{T}\left(Y_{t}-L_{t}\right) \mathrm{d} K_{t}=0$ and, since $Y_{t} \geq L_{t}$ and so

$$
\int_{0}^{T}\left(Y_{t}-L_{t}\right) \mathrm{d} K_{t} \geq 0
$$

it follows that $\int_{0}^{T}\left(Y_{t}-L_{t}\right) \mathrm{d} K_{t}=0$, i.e. that $(Y, Z, K)$ is the solution of $\operatorname{RBSDE}(\xi, f, L)$.

Step 3. In this step, we partly relax the assumption (2.4), which was widely used in Steps 1 and 2.

We now suppose only that

$$
\xi \geq c \quad \text { and } \inf _{0 \leq t \leq T} f(t, 0) \geq c,
$$

where $c$ is a constant. We approximate $\xi$ and $f(t, 0)$ each by a sequence whose elements satisfy the bounds assumed in Step 2, as follows: for each $n \in \mathbb{N}$, let

$$
\xi_{n}=\xi \wedge n, \quad f_{n}(t, y)=f(t, y)-f(t, 0)+f(t, 0) \wedge n .
$$

Obviously, $\left(\xi_{n}, f_{n}\right)$ satisfies the assumptions of Step 2 and, since $\xi \in \mathbf{L}^{2}\left(\mathcal{F}_{T}\right)$ and $f(t, 0) \in$ $\mathbf{H}^{2}(0, T)$,

$$
\mathrm{E}\left[\left|\xi_{n}-\xi\right|^{2}\right] \rightarrow 0 \quad \text { and } \quad \mathrm{E} \int_{0}^{T}\left|f(t, 0)-f_{n}(t, 0)\right|^{2} \mathrm{~d} t \rightarrow 0 \quad \text { as } n \rightarrow \infty .
$$

From the results in Step 2, for each $n \in \mathbb{N}$ there exists a triple

$$
\left(Y_{t}^{n}, Z_{t}^{n}, K_{t}^{n}\right)_{0 \leq t \leq T} \in \mathbf{S}^{2}(0, T) \times \mathbf{H}_{d}^{2}(0, T) \times \mathbf{A}^{2}(0, T)
$$

that is the unique solution of the $\operatorname{RBSDE}\left(\xi_{n}, f_{n}, L\right)$. By Theorem A.2, since $\xi_{n} \leq \xi_{n+1}$ and $f_{n}(s, y) \leq f_{n+1}(s, y)$ for all $(s, y) \in[0, T] \times \mathbb{R}$ and $n \in \mathbf{N}$, we have $Y_{t}^{n} \leq Y_{t}^{n+1}, 0 \leq t \leq T$, almost surely. Hence,

$$
Y_{t}^{n} \nearrow Y_{t}, \quad 0 \leq t \leq T, \quad \text { a.s. }
$$

Applying the Itô formula to $\left|Y_{t}^{n}-Y_{t}^{p}\right|^{2}$ for $n, p \in \mathbb{N}, n \geq p$, on the interval $[t, T]$, we find that

$$
\begin{aligned}
\mathrm{E}\left[\left|Y_{t}^{n}-Y_{t}^{p}\right|^{2}\right]+\mathrm{E} \int_{t}^{T}\left|Z_{s}^{n}-Z_{s}^{p}\right|^{2} \mathrm{~d} s \leq & \mathrm{E}\left[\left|\xi_{n}-\xi_{p}\right|^{2}\right]+\mathrm{E} \int_{t}^{T}\left|Y_{s}^{n}-Y_{s}^{p}\right|^{2} \mathrm{~d} s \\
& +\mathrm{E} \int_{t}^{T}\left|f_{n}(s, 0)-f_{p}(s, 0)\right|^{2} \mathrm{~d} s,
\end{aligned}
$$

since

$$
\begin{aligned}
\int_{t}^{T}\left(Y_{s}^{n}-Y_{s}^{p}\right) \mathrm{d}\left(K_{s}^{n}-K_{s}^{p}\right)= & \int_{t}^{T}\left(Y_{s}^{n}-L_{s}\right) \mathrm{d} K_{s}^{n}+\int_{t}^{T}\left(Y_{s}^{p}-L_{s}\right) \mathrm{d} K_{s}^{p} \\
& -\int_{t}^{T}\left(Y_{s}^{n}-L_{s}\right) \mathrm{d} K_{s}^{p}-\int_{t}^{T}\left(Y_{s}^{p}-L_{s}\right) \mathrm{d} K_{s}^{n} \\
\leq & 0 .
\end{aligned}
$$

Hence, from Gronwall's inequality and (2.34), we deduce that

$$
\sup _{0 \leq t \leq T} \mathrm{E}\left[\left|Y_{t}^{n}-Y_{t}^{p}\right|^{2}\right] \rightarrow 0, \quad \mathrm{E} \int_{0}^{T}\left|Z_{s}^{n}-Z_{s}^{p}\right|^{2} \mathrm{~d} s \rightarrow 0 .
$$


Consequently, there exists $\left(Z_{t}\right)_{0 \leq t \leq T} \in \mathbf{H}_{d}^{2}(0, T)$ such that

$$
\mathrm{E} \int_{0}^{T}\left|Z_{s}^{n}-Z_{s}\right|^{2} \mathrm{~d} s \rightarrow 0 .
$$

Using the Itô formula again, taking supremums and expectations, and in view of the BDG inequality, Assumption $2.2^{\prime \prime}\left(\mathrm{v}^{\prime \prime}\right)$, and the facts that $Y_{t}^{n} \geq Y_{t}^{p}$ and $f_{n}(t, 0) \geq f_{p}(t, 0)$, we find that

$$
\begin{aligned}
\mathrm{E}\left[\sup _{0 \leq t \leq T}\left|Y_{t}^{n}-Y_{t}^{p}\right|^{2}\right] \leq & \mathrm{E}\left[\left|\xi_{n}-\xi_{p}\right|^{2}\right]+2 \mathrm{E}\left[\sup _{0 \leq t \leq T} \int_{t}^{T}\left(Y_{s}^{n}-Y_{s}^{p}\right)\left(f_{n}(s, 0)-f_{p}(s, 0)\right) \mathrm{d} s\right] \\
& +\mathrm{E}\left[2 \sup _{0 \leq t \leq T}\left|\int_{t}^{T}\left(Y_{s}^{n}-Y_{s}^{p}\right)\left(Z_{s}^{n}-Z_{s}^{p}\right) \mathrm{d} B_{s}\right|\right] \\
\leq & \mathrm{E}\left[\left|\xi^{n}-\xi^{p}\right|\right]+4 T \mathrm{E} \int_{0}^{T}\left|f_{n}(s, 0)-f_{p}(s, 0)\right|^{2} \mathrm{~d} s \\
& +\frac{1}{4} \mathrm{E}\left[\sup _{0 \leq t \leq T}\left|Y_{s}^{n}-Y_{s}^{p}\right|^{2}\right]+\frac{1}{4} \mathrm{E}\left[\sup _{0 \leq t \leq T}\left|Y_{t}^{n}-Y_{t}^{p}\right|^{2}\right] \\
& +c \mathrm{E} \int_{0}^{T}\left|Z_{s}^{n}-Z_{s}^{p}\right|^{2} \mathrm{~d} s .
\end{aligned}
$$

From (2.34) and (2.36), it follows that $\mathrm{E}\left[\sup _{0 \leq t \leq T}\left|Y_{t}^{n}-Y_{t}^{p}\right|^{2}\right] \rightarrow 0$ as $n, p \rightarrow \infty$, i.e. the sequence $\left\{Y^{n}\right\}$ is a Cauchy sequence in the space $\mathbf{S}^{2}(0, T)$. Consequently, using (2.35), we have $Y \in \mathbf{S}^{2}(0, T)$ and

$$
\mathrm{E}\left[\sup _{0 \leq t \leq T}\left|Y_{t}^{n}-Y_{t}\right|^{2}\right] \rightarrow 0 .
$$

By Theorem A.4, since $\xi_{n} \leq \xi_{n+1}$ and $f_{n}(s, y) \leq f_{n+1}(s, y)$ for all $(s, y) \in[0, T] \times \mathbb{R}$ and $n \in \mathbb{N}$, we have $K_{t}^{n} \geq K_{t}^{n+1} \geq 0,0 \leq t \leq T$, and so

$$
K_{t}^{n} \searrow K_{t}
$$

with $\mathrm{E}\left[\left(K_{t}^{n}\right)^{2}\right]<\infty$. By the monotone limit theorem, it then follows that $K_{t}^{n} \rightarrow K_{t}$ in $\mathbf{L}^{2}\left(\mathcal{F}_{t}\right)$ with $\mathrm{E}\left[\left(K_{t}\right)^{2}\right]<\infty$, so $\left(K_{t}\right)_{0 \leq t \leq T}$ is increasing.

Notice that, since $f(t, y)$ is decreasing and continuous in $y$ and $Y_{t}^{n} \nearrow Y_{t}$, we have

$$
f\left(t, Y_{t}^{n}\right) \searrow f\left(t, Y_{t}\right) .
$$

Thus, by the monotone limit theorem, $\int_{0}^{t} f\left(s, Y_{s}^{n}\right) \mathrm{d} s \searrow \int_{0}^{t} f\left(s, Y_{s}\right) \mathrm{d} s$. Since $\left(Y^{n}, Z^{n}, K^{n}\right)$ is the solution of $\operatorname{RBSDE}\left(\xi^{n}, f_{n}, L\right)$, it also satisfies

$$
Y_{t}^{n}=Y_{0}^{n}-K_{t}^{n}-\int_{0}^{t} f\left(s, Y_{s}^{n}\right) \mathrm{d} s-\int_{0}^{t}\left(f_{n}(s, 0)-f(s, 0)\right) \mathrm{d} s+\int_{0}^{t} Z_{s}^{n} \mathrm{~d} B_{s},
$$

and, using (2.34), (2.35), (2.37), and (2.38), we find that $(Y, Z, K)$ satisfies

$$
Y_{t}=Y_{0}-K_{t}-\int_{0}^{t} f\left(s, Y_{s}\right) \mathrm{d} s+\int_{0}^{t} Z_{s} \mathrm{~d} B_{s} .
$$

Therefore,

$$
Y_{t}=\xi+\int_{t}^{T} f\left(s, Y_{s}\right) \mathrm{d} s+K_{T}-K_{t}-\int_{t}^{T} Z_{s} \mathrm{~d} B_{S}
$$


Since $\left(Y_{t}^{n}, Z_{t}^{n}, K_{t}^{n}\right)_{0 \leq t \leq T} \in \mathbf{S}^{2}(0, T) \times \mathbf{H}_{d}^{2}(0, T) \times \mathbf{A}^{2}(0, T)$, we have, for $0 \leq t \leq T$,

$$
\mathrm{E}\left[\left(\int_{0}^{t} f_{n}\left(s, Y_{s}^{n}\right) \mathrm{d} s\right)^{2}\right] \leq 2 \mathrm{E}\left[\left(Y_{t}^{n}\right)^{2}\right]+2\left(Y_{0}^{n}\right)^{2}+2 \mathrm{E}\left[\left(K_{t}^{n}\right)^{2}\right]+2 \mathrm{E} \int_{0}^{t}\left(Z_{s}^{n}\right)^{2} \mathrm{~d} s<\infty .
$$

From the definition of $f_{n}(s, y)$, it follows that, for $n \in \mathbb{N}$,

$$
\begin{aligned}
\mathrm{E}\left[\left(\int_{0}^{t} f\left(s, Y_{s}^{n}\right) \mathrm{d} s\right)^{2}\right] & \leq 2 \mathrm{E}\left[\left(\int_{0}^{t} f_{n}\left(s, Y_{s}^{n}\right) \mathrm{d} s\right)^{2}\right]+2 \mathrm{E}\left[\left(\int_{0}^{t}\left(f(s, 0)-f_{n}(s, 0)\right) \mathrm{d} s\right)^{2}\right] \\
& <\infty .
\end{aligned}
$$

Then, from (2.40),

$$
\mathrm{E}\left[\left(\int_{0}^{t} f\left(s, Y_{s}\right) \mathrm{d} s\right)^{2}\right] \leq 2 \mathrm{E}\left[\left(Y_{t}\right)^{2}\right]+2\left(Y_{0}\right)^{2}+2 \mathrm{E}\left[\left(K_{t}\right)^{2}\right]+2 \mathrm{E} \int_{0}^{t}\left(Z_{S}\right)^{2} \mathrm{~d} s<\infty
$$

for $0 \leq t \leq T$, and it follows that $\int_{0}^{t} f\left(s, Y_{s}^{n}\right) \mathrm{d} s \rightarrow \int_{0}^{t} f\left(s, Y_{s}\right) \mathrm{d} s$ in $\mathbf{L}^{2}\left(\mathcal{F}_{t}\right)$ as $n \rightarrow \infty$.

Now we must prove that the convergence of $\left\{K^{n}\right\}$ holds in a stronger sense. Let us rewrite (2.39) and (2.40) in forward form (as in (2.12)), and consider their difference. By the BDG inequality and the facts that $f\left(s, Y_{s}^{n}\right) \geq f\left(s, Y_{s}\right)$ and $f(s, 0) \geq f_{n}(s, 0)$, we deduce that

$$
\begin{aligned}
\mathrm{E}\left[\sup _{0 \leq t \leq T}\left|K_{t}^{n}-K_{t}\right|^{2}\right] \\
\leq 2\left|Y_{0}^{n}-Y_{0}\right|^{2}+2 \mathrm{E}\left[\sup _{0 \leq t \leq T}\left|Y_{t}^{n}-Y_{t}\right|^{2}\right]+2 \mathrm{E}\left[\left(\int_{0}^{T} f\left(s, Y_{s}^{n}\right)-f\left(s, Y_{s}\right) \mathrm{d} s\right)^{2}\right] \\
\quad+2 \mathrm{E}\left[\left(\int_{0}^{T} f(s, 0)-f_{n}(s, 0) \mathrm{d} s\right)^{2}\right]+2 \mathrm{E} \int_{0}^{t}\left(Z_{s}^{n}-Z_{s}\right)^{2} \mathrm{~d} s .
\end{aligned}
$$

It follows that $\mathrm{E}\left[\sup _{0 \leq t \leq T}\left|K_{t}^{n}-K_{t}\right|^{2}\right] \rightarrow 0$ as $n \rightarrow 0$, and the convergence holds in $\mathbf{S}^{2}(0, T)$.

It remains to check that $\left(Y_{t}, Z_{t}, K_{t}\right)_{0 \leq t \leq T}$ satisfies properties 3 and 4 of Definition 2.1. Since $Y_{t}^{n} \geq L_{t}$ and $0 \leq t \leq T$, we have $Y_{t} \geq L_{t}, 0 \leq t \leq T$, almost surely. Furthermore, $\left(Y^{n}, K^{n}\right)$ tends to $(Y, K)$ uniformly in $t$ in probability, as $n \rightarrow \infty$. Therefore, as at the end of Step 1, we conclude that $\int_{0}^{T}\left(Y_{t}-L_{t}\right) \mathrm{d} K_{t}=0$, i.e. the triple $\left(Y_{t}, Z_{t}, K_{t}\right)_{0 \leq t \leq T}$ is the solution of $\operatorname{RBSDE}(\xi, f, L)$ under the assumption (2.33).

Step 4. Now we consider a terminal condition $\xi \in \mathbf{L}^{2}\left(\mathcal{F}_{T}\right)$ and a coefficient $f$ that satisfies Assumption 2.2", as follows:

$$
\xi_{n}=\xi \vee(-n) \quad \text { and } \quad f_{n}(t, y)=f(t, y)-f(t, 0)+f(t, 0) \vee(-n)
$$

for $n \in \mathbb{N}$. It is clear that $\xi_{n}$ and $f_{n}$ satisfy the assumptions of Step 3, and that

$$
\mathrm{E}\left[\left|\xi_{n}-\xi\right|^{2}\right] \rightarrow 0, \quad \mathrm{E} \int_{0}^{T}\left|f(t, 0)-f_{n}(t, 0)\right|^{2} \mathrm{~d} t \rightarrow 0 .
$$

By the results of Step 3, there exists a triple

$$
\left(Y_{t}^{n}, Z_{t}^{n}, K_{t}^{n}\right)_{0 \leq t \leq T} \in \mathbf{S}^{2}(0, T) \times \mathbf{H}_{d}^{2}(0, T) \times \mathbf{A}^{2}(0, T)
$$


that is the solution of the $\operatorname{RBSDE}\left(\xi_{n}, f_{n}, L\right)$. By Theorem A.2, as $n \rightarrow \infty, Y_{t}^{n} \searrow Y_{t}$ for $0 \leq t \leq T$ almost surely and, as in Step 3, we find that $\left(Y_{t}^{n}\right)_{0 \leq t \leq T} \rightarrow\left(Y_{t}\right)_{0 \leq t \leq T}$ in $\mathbf{S}^{2}(0, T)$ and $\left(Z_{t}^{n}\right)_{0 \leq t \leq T} \rightarrow\left(Z_{t}\right)_{0 \leq t \leq T}$ in $\mathbf{H}_{d}^{2}(0, T)$.

Now we must prove the convergence of $\left(K_{t}^{n}\right)_{0 \leq t \leq T}$. Set $\xi_{n, m}=\xi_{n} \wedge m=(\xi \vee(-n)) \wedge m$ and

$$
\begin{aligned}
f_{n, m}(t, y) & =f_{n}(t, y)-f_{n}(t, 0)+f_{n}(t, 0) \wedge m \\
& =f(t, y)-f(t, 0)+(f(t, 0) \vee(-n)) \wedge m,
\end{aligned}
$$

for $m \in \mathbb{N}$. Then, $\left|\xi_{n, m}\right|+\sup _{0 \leq t \leq T}\left|f_{n, m}(t, 0)\right| \leq c, \xi_{n, m} \geq \xi_{n+1, m}$, and $f_{n, m}(t, y) \geq$ $f_{n+1, m}(t, y)$. From Theorem A.4, considering the solutions $\left(Y_{t}^{m, n}, Z_{t}^{m, n}, K_{t}^{m, n}\right)_{0 \leq t \leq T}$ to the $\operatorname{RBSDEs}\left(\xi^{m, n}, f_{m, n}, L\right)$, we find that $K_{t}^{m, n} \leq K_{t}^{m, n+1}$ for $t \in[0, T]$. Due to the convergence results in Step 3, we know that $K_{t}^{m, n} \rightarrow K_{t}^{n}$ and $K_{t}^{m, n+1} \rightarrow K_{t}^{n+1}$ in $\mathbf{L}^{2}\left(\mathcal{F}_{t}\right)$ as $m \rightarrow \infty$. Therefore $K_{t}^{n} \leq K_{t}^{n+1}$ for $t \in[0, T]$, with $\mathrm{E}\left[\left(K_{t}^{n}\right)^{2}\right]<\infty$, and, by the monotone limit theorem, it follows that $K_{t}^{n} \nearrow K_{t}$ in $\mathbf{L}^{2}\left(\mathcal{F}_{t}\right)$.

So, by the same method as in Step 3, we deduce that the limit

$$
\left(Y_{t}, Z_{t}, K_{t}\right)_{0 \leq t \leq T} \in \mathbf{S}^{2}(0, T) \times \mathbf{H}_{d}^{2}(0, T) \times \mathbf{A}^{2}(0, T)
$$

is the solution of the $\operatorname{RBSDE}(\xi, f, L)$.

This completes the proof of Theorem 2.2, and we conclude that, under Assumptions 2.1, $2.2^{\prime}$, and $2.3^{\prime}$, the $\operatorname{RBSDE}(\xi, f, L)$ has a unique solution $\left(Y_{t}, Z_{t}, K_{t}\right)_{0 \leq t \leq T}$.

\section{Application to finance}

We follow the idea of El Karoui et al. (1997b). In some constraint cases we consider the strategy wealth portfolio $\left(X_{t}, \pi_{t}\right)$ as a pair of adapted processes in $\mathbf{H}^{2}(0, T) \times \mathbf{H}_{d}^{2}(0, T)$ that satisfy the following BSDE:

$$
-\mathrm{d} X_{t}=b\left(t, X_{t}, \pi_{t}\right) \mathrm{d} t-\pi_{t}^{\top} \sigma_{t} \mathrm{~d} B_{t},
$$

where $b$ is $\mathbb{R}$-valued, convex with respect to $(x, \pi)$, and satisfies Assumption 2.2. We suppose that the volatility matrix $\sigma$ of the $n$ risky assets is invertible and such that $\left(\sigma_{t}\right)^{-1}$ is bounded. Without loss of generality, we take $\sigma_{t}=I_{d}$, the $d$-dimensional identity matrix.

We are concerned with the problem of pricing, at each time $t$, an American contingent claim, which consists of the selection of a stopping time $\tau \in \mathcal{T}_{t}$ (the set of stopping times valued in $[t, T]$ ) and a payoff on exercise $S_{\tau}$ if $\tau<T$ and $\xi$ if $\tau=T$. Here, $\left(S_{t}\right)$ satisfies Assumption 2.3. We set

$$
\tilde{S}_{s}=\xi \mathbf{1}_{\{s=T\}}+S_{s} \mathbf{1}_{\{s<T\}},
$$

and fix $t \in[0, T]$ and $\tau \in \mathcal{T}_{t}$. Then (Pardoux 1999), there exists a unique strategy

$$
\left(X_{S}\left(\tau, \tilde{S}_{\tau}\right), \pi\left(\tau, \tilde{S}_{\tau}\right)\right) \in \mathbf{H}^{2}(0, T) \times \mathbf{H}_{d}^{2}(0, T),
$$

denoted by $\left(X_{s}^{\tau}, \pi_{s}^{\tau}\right)$, which replicates $\tilde{S}_{\tau}$, i.e. is the solution of the classical BSDE associated with terminal time $\tau$, terminal condition $\tilde{S}_{\tau}$, and generator $b$ :

$$
\begin{aligned}
-\mathrm{d} X_{s}^{\tau} & =b\left(s, X_{s}^{\tau}, \pi_{s}^{\tau}\right) \mathrm{d} s-\left(\pi_{s}^{\tau}\right)^{\top} \mathrm{d} B_{s}, \quad 0 \leq s \leq T, \\
X_{\tau}^{\tau} & =\tilde{S}_{\tau} .
\end{aligned}
$$


Therefore, the price of the American contingent claim $\left(\tilde{S}_{s}, 0 \leq s \leq T\right)$ at time $t$ is given by

$$
X_{t}=\underset{\tau \in \mathcal{T}_{t}}{\operatorname{ess} \sup } X_{t}\left(\tau, \tilde{S}_{\tau}\right)
$$

Applying the previous results on RBSDEs, it follows that the price $\left(X_{t}, 0 \leq t \leq T\right)$ corresponds to the unique solution of the RBSDE associated with terminal condition $\xi$, coefficient $b$, and barrier $S$, i.e. there exist $\left(\pi_{t}\right) \in \mathbf{H}_{d}^{2}(0, T)$ and $\left(K_{t}\right)$, an increasing, adapted, continuous process with $K_{0}=0$, such that

$$
\begin{aligned}
-\mathrm{d} X_{t} & =b\left(s, X_{t}, \pi_{t}\right) \mathrm{d} s+\mathrm{d} K_{t}-\pi_{t}^{\top} \mathrm{d} B_{t}, \\
X_{T} & =\xi, \\
X_{t} & \geq S_{t}, \quad 0 \leq t \leq T, \quad \int_{0}^{T}\left(X_{t}-S_{t}\right) \mathrm{d} K_{t}=0 .
\end{aligned}
$$

Furthermore, the stopping time $D_{t}=\inf \left(t \leq s \leq T \mid X_{s}=S_{S}\right) \wedge T$ is optimal, that is,

$$
X_{t}=X_{t}\left(D_{t}, \tilde{S}_{D_{t}}\right) \text {. }
$$

\section{Appendix A.}

In this section, we present several comparison theorems, which are used in the proof of Theorem 2.2. The first is a generalized version of the comparison theorem in Pardoux (1999), which treats the one-dimensional case.

Theorem A.1. (General case for BSDEs.) Suppose that $f^{1}(s, y, z)$ and $f^{2}(s, y, z)$ satisfy Assumption 2.2, that $\xi^{1}, \xi^{2} \in \mathbf{L}^{2}\left(\mathcal{F}_{T}\right)$, and that $K^{1}$ and $K^{2}$ are two continuous, increasing processes with $\mathrm{E}\left[\left(K_{T}^{i}\right)^{2}\right] \leq c, i=1$, 2. If there exist pairs $\left(Y_{t}^{i}, Z_{t}^{i}\right)_{0 \leq t \leq T}, i=1,2$, satisfying the equations

$$
Y_{t}^{i}=\xi^{i}+\int_{t}^{T} f^{i}\left(s, Y_{s}^{i}, Z_{s}^{i}\right) \mathrm{d} s+K_{T}^{i}-K_{t}^{i}-\int_{t}^{T} Z_{s}^{i} \mathrm{~d} B_{s}, \quad i=1,2,
$$

and, moreover, if for any $0 \leq t \leq T$,

$$
f^{1}\left(t, Y_{t}^{1}, Z_{t}^{1}\right) \leq f^{2}\left(t, Y_{t}^{1}, Z_{t}^{1}\right), \quad \xi^{1} \leq \xi^{2},
$$

and $K^{2}-K^{1}$ is an increasing process, then $Y_{t}^{1} \leq Y_{t}^{2}, 0 \leq t \leq T$, almost surely.

Proof. Define

$$
\begin{aligned}
& \alpha_{t}= \begin{cases}\frac{f^{2}\left(t, Y_{t}^{2}, Z_{t}^{2}\right)-f^{2}\left(t, Y_{t}^{1}, Z_{t}^{2}\right)}{Y_{t}^{2}-Y_{t}^{1}} & \text { if } Y_{t}^{2} \neq Y_{t}^{1}, \\
0 & \text { if } Y_{t}^{2}=Y_{t}^{1},\end{cases} \\
& \beta_{t}^{i}= \begin{cases}\frac{f^{2}\left(t, Y_{t}^{1}, \tilde{Z}_{t}^{i-1}\right)-f^{2}\left(t, Y_{t}^{1}, \tilde{Z}_{t}^{i}\right)}{Z_{t}^{2, i}-Z_{t}^{1, i}} & \text { if } Z_{t}^{2, i} \neq Z_{t}^{1, i}, \\
0 & \text { if } Z_{t}^{2, i}=Z_{t}^{1, i},\end{cases}
\end{aligned}
$$

where $i=1, \ldots, d$. Here $\tilde{Z}_{t}^{i}$ is the vector whose first $i$ components are equal to those of $Z_{t}^{1}$ and whose last $d-i$ components are equal to those of $Z_{t}^{2}$, that is,

$$
\tilde{Z}_{t}^{i}=\left(Z_{t}^{1,1}, \ldots, Z_{t}^{1, i}, Z_{t}^{2, i+1}, \ldots, Z_{t}^{2, d}\right) .
$$


Obviously, $\alpha_{t}$ and $\beta_{t}$ are progressively measurable and, by parts (iv) and (v) of Assumption 2.2, $\alpha_{t} \leq \mu$ and $\left|\beta_{t}\right| \leq C$.

For $0 \leq s \leq t \leq T$, let $\Gamma_{s, t}=\exp \left[\int_{s}^{t}\left(\alpha_{r}-\frac{1}{2}\left|\beta_{r}\right|^{2}\right) \mathrm{d} r+\int_{s}^{t} \beta_{r} \mathrm{~d} B_{r}\right]$. Consider the difference of the two solutions of the BSDEs, i.e. $\Delta Y_{t}=Y_{t}^{2}-Y_{t}^{1}$ and $\Delta Z_{t}=Z_{t}^{2}-Z_{t}^{1}$, and $\Delta \xi=\xi^{2}-\xi^{1}, U_{t}=f^{2}\left(t, Y_{t}^{1}, Z_{t}^{1}\right)-f^{1}\left(t, Y_{t}^{1}, Z_{t}^{1}\right)$, and $\Delta K_{t}=K_{t}^{2}-K_{t}^{1}$; of these, we know that $\Delta \xi \geq 0, U_{t} \geq 0$, and $\mathrm{d}\left(\Delta K_{t}\right) \geq 0$.

The pair $\left(\Delta Y_{t}, \Delta Z_{t}\right)$ solves the equation

$$
\Delta Y_{t}=\Delta \xi+\int_{t}^{T}\left(\alpha_{s} \Delta Y_{s}+\beta_{s} \Delta Z_{s}\right) \mathrm{d} s+\int_{t}^{T} U_{s} \mathrm{~d} s+\Delta K_{T}-\Delta K_{t}-\int_{t}^{T} \Delta Z_{s} \mathrm{~d} B_{s} .
$$

Applying the Itô formula to $\Delta Y_{S} \Gamma_{s, t}$, we find that

$$
\Delta Y_{s}=\Gamma_{s, t} \Delta Y_{t}+\int_{s}^{t} \Gamma_{s, r} U_{r} \mathrm{~d} r+\int_{s}^{t} \Gamma_{s, r} \mathrm{~d}\left(\Delta K_{r}\right)-\int_{s}^{t} \Gamma_{s, r}\left(\Delta Z_{r}+\Delta Y_{r} \beta_{r}\right) \mathrm{d} B_{r},
$$

so, taking the conditional expectation, it follows that

$$
\Delta Y_{s}=\mathrm{E}\left[\Gamma_{s, t} \Delta Y_{t}+\int_{s}^{t} \Gamma_{s, r} U_{r} \mathrm{~d} r+\int_{s}^{t} \Gamma_{s, r} \mathrm{~d}\left(\Delta K_{r}\right) \mid \mathcal{F}_{s}\right]
$$

In particular,

$$
\Delta Y_{t}=\mathrm{E}\left[\Gamma_{t, T} \Delta \xi+\int_{t}^{T} \Gamma_{t, r} U_{r} \mathrm{~d} r+\int_{t}^{T} \Gamma_{t, r} \mathrm{~d}\left(\Delta K_{r}\right) \mid \mathcal{F}_{t}\right] \geq 0
$$

using the positivity of $\Delta \xi, U$, and $\Delta K$.

We next prove a comparison theorem for the solution of the RBSDE in the general case, which is similar to that in El Karoui et al. (1997a).

Theorem A.2. (General case for RBSDEs.) Suppose that the parameters $\left(\xi^{1}, f^{1}, L^{1}\right)$ and $\left(\xi^{2}, f^{2}, L^{2}\right)$ satisfy Assumptions 2.1, 2.2, and 2.3. Let $\left(Y_{t}^{i}, Z_{t}^{i}, K_{t}^{i}\right)_{0 \leq t \leq T}$ be the solution of the $\operatorname{RBSDE}\left(\xi^{i}, f^{i}, L^{i}\right), i=1,2$. Also assume the following:

$$
\begin{aligned}
\xi^{1} & \leq \xi^{2} \quad \text { a.s. } \\
f^{1}(t, y, z) & \leq f^{2}(t, y, z) \quad \text { for all }(t, y) \in[0, T] \times \mathbb{R}, \text { a.s. } \\
L_{t}^{1} & \leq L_{t}^{2} \quad \text { for all } t \in[0, T], \text { a.s. }
\end{aligned}
$$

Then $Y_{t}^{1} \leq Y_{t}^{2}$ for $t \in[0, T]$, almost surely.

Proof. Applying the Itô formula to $\left(\left(Y^{1}-Y^{2}\right)^{+}\right)^{2}$ on the interval $[t, T]$, and taking expectations on both sides, we find that

$$
\begin{aligned}
\mathrm{E}\left[\left(\left(Y_{t}^{1}-Y_{t}^{2}\right)^{+}\right)^{2}\right]+\mathrm{E} \int_{t}^{T} \mathbf{1}_{\left\{Y_{t}^{1}>Y_{t}^{2}\right\}}\left|Z_{s}^{1}-Z_{s}^{2}\right|^{2} \mathrm{~d} s \\
=\mathrm{E}\left[\left(\xi^{1}-\xi^{2}\right)^{+}\right]+2 \mathrm{E} \int_{t}^{T}\left(Y_{s}^{1}-Y_{s}^{2}\right)^{+} \mathbf{1}_{\left\{Y_{s}^{1}>Y_{s}^{2}\right\}}\left(f^{1}\left(s, Y_{s}^{1}, Z_{s}^{1}\right)-f^{2}\left(s, Y_{s}^{2}, Z_{s}^{2}\right)\right) \mathrm{d} s \\
\quad+2 \mathrm{E} \int_{t}^{T}\left(Y_{s}^{1}-Y_{s}^{2}\right)^{+} \mathrm{d}\left(K_{s}^{1}-K_{s}^{2}\right) .
\end{aligned}
$$


Since $Y_{t}^{1}>Y_{t}^{2} \geq L_{t}^{2} \geq L_{t}^{1}$ on the set $\left\{Y_{t}^{1}>Y_{t}^{2}\right\}$, we have

$$
\begin{aligned}
\int_{t}^{T}\left(Y_{s}^{1}-Y_{s}^{2}\right)^{+} \mathrm{d}\left(K_{s}^{1}-K_{s}^{2}\right) \leq & \int_{t}^{T}\left(Y_{s}^{1}-L_{s}\right) \mathrm{d} K_{s}^{1}-\int_{t}^{T}\left(Y_{s}^{2}-L_{s}\right) \mathrm{d} K_{s}^{1} \\
& -\int_{t}^{T}\left(Y_{s}^{1}-Y_{s}^{2}\right)^{+} \mathrm{d} K_{s}^{2} \\
\leq & -\int_{t}^{T}\left(Y_{s}^{1}-Y_{s}^{2}\right)^{+} \mathrm{d} K_{s}^{2} \leq 0
\end{aligned}
$$

so, by (A.1) and the Lipschitz and monotonicity conditions on $f^{2}$, it follows that

$$
\begin{aligned}
& \mathrm{E}\left[\left(\left(Y_{t}^{1}-\right.\right.\right.\left.\left.\left.Y_{t}^{2}\right)^{+}\right)^{2}\right]+\mathrm{E} \int_{t}^{T} \mathbf{1}_{\left\{Y_{t}^{1}>Y_{t}^{2}\right\}}\left|Z_{s}^{1}-Z_{s}^{2}\right|^{2} \mathrm{~d} s \\
& \leq 2 \mathrm{E} \int_{t}^{T} \mathbf{1}_{\left\{Y_{s}^{1}>Y_{s}^{2}\right\}}\left(Y_{s}^{1}-Y_{s}^{2}\right)\left(f^{1}\left(s, Y_{s}^{1}, Z_{s}^{1}\right)-f^{2}\left(s, Y_{s}^{1}, Z_{s}^{1}\right)\right.\left.\quad+f^{2}\left(s, Y_{s}^{1}, Z_{s}^{1}\right)-f^{2}\left(s, Y_{s}^{2}, Z_{s}^{2}\right)\right) \mathrm{d} s \\
& \leq 2 \mathrm{E} \int_{t}^{T} \mathbf{1}_{\left\{Y_{s}^{1}>Y_{s}^{2}\right\}}\left(Y_{s}^{1}-Y_{s}^{2}\right)\left(f^{2}\left(s, Y_{s}^{1}, Z_{s}^{1}\right)-f^{2}\left(s, Y_{s}^{2}, Z_{s}^{2}\right)\right) \mathrm{d} s \\
& \leq \frac{1}{2} \mathrm{E} \int_{t}^{T} \mathbf{1}_{\left\{Y_{t}^{1}>Y_{t}^{2}\right\}}\left|Z_{s}^{1}-Z_{s}^{2}\right|^{2} \mathrm{~d} s+\left(2 \mu+4 C^{2}\right) \mathrm{E} \int_{t}^{T}\left[\left(Y_{s}^{1}-Y_{s}^{2}\right)^{+}\right]^{2} \mathrm{~d} s
\end{aligned}
$$

Hence,

$$
\mathrm{E}\left[\left(\left(Y_{t}^{1}-Y_{t}^{2}\right)^{+}\right)^{2}\right] \leq\left(2 \mu+4 C^{2}\right) \mathrm{E} \int_{t}^{T}\left[\left(Y_{s}^{1}-Y_{s}^{2}\right)^{+}\right]^{2} \mathrm{~d} s
$$

and, from Gronwall's inequality, we deduce that $\left(Y_{t}^{1}-Y_{t}^{2}\right)^{+}=0,0 \leq t \leq T$.

In this comparison theorem, we can only compare the two solutions $Y^{i}$ of the RBSDEs with different coefficients; since the barriers $L^{1}$ and $L^{2}$ are different, we cannot compare the increasing processes of the two solutions. However, the following comparison theorem shows that if the two barriers are the same and satisfy Assumption 2.3, then we can compare the increasing processes $K^{i}$. In the following we first prove a comparison theorem under the boundedness condition for $\xi, f$, and $\sup _{0 \leq t \leq T} L_{t}^{+}$, and then relax it step by step.

Theorem A.3. (Special case for RBSDEs.) Suppose that $f^{1}(s, y)$ and $f^{2}(s, y)$ satisfy Assumption 2.2" , and that $L, \xi^{i}$, and $f^{i}(\cdot, 0), i=1,2$, satisfy

$$
\left|\xi^{i}\right|+\sup _{0 \leq t \leq T}\left|f^{i}(t, 0)\right|+\sup _{0 \leq t \leq T} L_{t}^{+} \leq c, \quad i=1,2
$$

for some constant c. Denote by $\left(Y_{t}^{i}, Z_{t}^{i}, K_{t}^{i}\right)_{0 \leq t \leq T}$ the solution of the $\operatorname{RBSDE}\left(\xi^{i}, f^{i}, L\right)$, $i=1$, 2. If we have

$$
\begin{aligned}
f^{1}(t, y) & \leq f^{2}(t, y) \quad \text { for all }(t, y) \in[0, T] \times \mathbb{R}, \text { a.s. }, \\
\xi^{1} & \leq \xi^{2} \quad \text { a.s. }
\end{aligned}
$$

then $Y_{t}^{1} \leq Y_{t}^{2}$ and $K_{t}^{1} \geq K_{t}^{2}$ for $t \in[0, T]$, almost surely, and, for $0 \leq s \leq t \leq T$, $K_{t}^{1}-K_{s}^{1} \geq K_{t}^{2}-K_{s}^{2}$ almost surely. 
Proof. We consider the penalized equations relative to the $\operatorname{RBSDE}\left(\xi^{i}, f^{i}, L\right)$, for $i=1,2$ and $n \in \mathbb{N}$, as follows:

$$
Y_{t}^{n, i}=\xi^{i}+\int_{t}^{T} f^{i}\left(s, Y_{s}^{n, i}\right) \mathrm{d} s+n \int_{t}^{T}\left(Y_{s}^{n, i}-L_{s}\right)^{-} \mathrm{d} s-\int_{t}^{T} Z_{s}^{n, i} \mathrm{~d} B_{s} .
$$

For each $n \in \mathbb{N}$,

$$
f_{n}^{1}(s, y):=f^{1}(s, y)+n\left(y-L_{s}\right)^{-} \leq f_{n}^{2}(s, y):=f^{2}(s, y)+n\left(y-L_{s}\right)^{-},
$$

so, by the comparison theorem in Pardoux (1999), we have

$$
Y_{t}^{n, 1} \leq Y_{t}^{n, 2}, \quad 0 \leq t \leq T .
$$

Since $K_{t}^{n, i}=n \int_{0}^{t}\left(Y_{s}^{n, i}-L_{s}\right)^{-} \mathrm{d} s$, we deduce that, for $0 \leq s \leq t \leq T$,

$$
\begin{aligned}
K_{t}^{n, 1} & \geq K_{t}^{n, 2}, \\
K_{t}^{n, 1}-K_{s}^{n, 1} & \geq K_{t}^{n, 2}-K_{s}^{n, 2} .
\end{aligned}
$$

Since $Y_{t}^{n, 1} \nearrow Y_{t}^{1}, Y_{t}^{n, 2} \nearrow Y_{t}^{2}, K_{t}^{n, 1} \rightarrow K_{t}^{1}$, and $K_{t}^{n, 2} \rightarrow K_{t}^{2}$ almost surely, by the convergence results of Step 1 the inequalities

$$
Y_{t}^{1} \leq Y_{t}^{2}, \quad K_{t}^{1} \geq K_{t}^{2}, \quad \text { and } \quad K_{t}^{1}-K_{s}^{1} \geq K_{t}^{2}-K_{s}^{2}
$$

hold for $0 \leq s \leq t \leq T$.

In the next theorem, we relax the boundedness condition on the barrier $L^{+}$.

Theorem A.4. (Special case for RBSDEs.) Suppose that $f^{1}(s, y)$ and $f^{2}(s, y)$ satisfy Assumption 2.2", that the barrier L satisfies Assumption 2.3' ${ }^{\prime}$ and that $\xi^{i}$ and $f^{i}(\cdot, 0), i=1,2$, satisfy

$$
\left|\xi^{i}\right|+\sup _{0 \leq t \leq T}\left|f^{i}(t, 0)\right| \leq c
$$

for some constant c. Denote by $\left(Y_{t}^{i}, Z_{t}^{i}, K_{t}^{i}\right)_{0 \leq t \leq T}$ the solution of the $\operatorname{RBSDE}\left(\xi^{i}, f^{i}, L\right)$, $i=1$, 2. If we have

$$
\begin{aligned}
f^{1}(t, y) & \leq f^{2}(t, y) \quad \text { for all }(t, y) \in[0, T] \times \mathbb{R}, \text { a.s. } \\
\xi^{1} & \leq \xi^{2} \quad \text { a.s. }
\end{aligned}
$$

then $Y_{t}^{1} \leq Y_{t}^{2}$ and $K_{t}^{1} \geq K_{t}^{2}$ for $t \in[0, T]$, almost surely, and, for $0 \leq s \leq t \leq T$, $K_{t}^{1}-K_{s}^{1} \geq K_{t}^{2}-K_{s}^{2}$ almost surely.

Proof. As in Step 2, there exist constants $c_{1}$ and $c_{2}$ such that $\xi^{i} \leq c_{1}$ and $f^{i}(t, 0) \leq c_{2}$ for $i=1,2$; set $c^{\prime}=\max \left\{c_{1}, c_{2} T\right\}$. Then, for $i=1,2,\left(Y_{t}^{i}, Z_{t}^{i}, K_{t}^{i}\right)_{0 \leq t \leq T}$ is the solution of the $\operatorname{RBSDE}\left(\xi^{i}, f^{i}, L\right)$ if and only if $\left(Y_{t}^{i \prime}, Z_{t}^{i \prime}, K_{t}^{i \prime}\right)_{0 \leq t \leq T}$ is the solution of the $\operatorname{RBSDE}\left(\xi^{i \prime}, f^{i \prime}, L^{\prime}\right)$, where

$$
\left(Y_{t}^{i \prime}, Z_{t}^{i \prime}, K_{t}^{i \prime}\right)=\left(Y_{t}^{i}+c_{2} t-2 c^{\prime}, Z_{t}^{i}, K_{t}^{i}\right)
$$

and

$$
\left(\xi^{i \prime}, f^{i \prime}(t, y), L_{t}^{\prime}\right)=\left(\xi^{i}+c_{2} T-2 c^{\prime}, f^{i}\left(t, y-\left(c_{2} t-2 c^{\prime}\right)\right)-c_{2}, L_{t}+c_{2} t-2 c^{\prime}\right) .
$$


From this transformation, we know that, for $0 \leq s \leq t \leq T$, the result is equivalent to

$$
Y_{t}^{1 \prime} \leq Y_{t}^{2 \prime}, \quad K_{t}^{1 \prime} \geq K_{t}^{2 \prime}, \quad K_{t}^{1 \prime}-K_{s}^{1 \prime} \geq K_{t}^{2 \prime}-K_{s}^{2 \prime}, \quad \text { a.s. }
$$

Setting $L^{n}=L^{\prime} \wedge n$, we consider the solution

$$
\left(Y_{t}^{n, i \prime}, Z_{t}^{n, i \prime}, K_{t}^{n, i \prime}\right)_{0 \leq t \leq T}
$$

of the $\operatorname{RBSDE}\left(\xi^{i \prime}, f^{i \prime}, L^{n}\right)$, since $\sup _{0 \leq t \leq T}\left(L_{t}^{n}\right)^{+} \leq c, \xi^{1 \prime} \leq \xi^{2 \prime}$, and

$$
f^{1 \prime}(t, y) \leq f^{2 \prime}(t, y) \quad \text { for all }(t, y) \in[0, T] \times \mathbb{R} .
$$

Then, by Theorem A.3, we have

$$
Y_{t}^{n, 1 \prime} \leq Y_{t}^{n, 2 \prime}, \quad K_{t}^{n, 1 \prime} \geq K_{t}^{n, 2 \prime}, \quad K_{t}^{n, 1 \prime}-K_{s}^{n, 1 \prime} \geq K_{t}^{n, 2 \prime}-K_{s}^{n, 2 \prime}, \quad \text { a.s., }
$$

for $0 \leq s \leq t \leq T$. Notice that $\xi^{i \prime} \leq 0$ and $f^{i \prime}(t, 0) \leq 0$ for $i=1,2$; by the proof of Lemma 2.3, we deduce that $Y_{t}^{n, i \prime} \nearrow Y_{t}^{\overline{i \prime}}$ almost surely and that $K_{t}^{n, i \prime} \rightarrow K_{t}^{i \prime}$ in $\mathbf{L}^{2}\left(\mathcal{F}_{t}\right)$, for $0 \leq t \leq T$ and $i=1,2$. So, letting $n \rightarrow \infty$, (A.2) follows and the proof is complete.

\section{References}

Briand, Ph. And Carmona, R. (2000). BSDEs with polynomial growth generators. J. Appl. Math. Stoch. Anal. 13, 207-238.

Briand, Ph. et al. (2003). $L_{p}$ solutions of backward stochastic differential equations. Stoch. Process. Appl. 108, 109-129.

Duffie, D. And Epstein, L. (1992). Stochastic differential utility. Econometrica 60, 353-394.

El Karoui, N. et al. (1997a). Reflected solutions of backward SDE's, and related obstacle problems for PDE's. Ann. Prob. 25, 702-737.

El Karoui, N., Pardoux, E. And Quenez, M. C. (1997b). Reflected backward SDEs and American options. In Numerical Methods in Finance, Cambridge University Press, pp. 215-231.

Matoussi, A. (1997). Reflected solutions of backward stochastic differential equations with continuous coefficient. Statist. Prob. Lett. 34, 347-354.

PARdoux, E. (1999). BSDEs, weak convergence and homogenization of semilinear PDEs. In Nonlinear Analysis, Differential Equations and Control, eds F. H. Clarke and R. J. Stern, Kluwer, Dordrecht, pp. 503-549.

Pardoux, E. ANd Peng, S. G. (1990). Adapted solution of a backward stochastic differential equation. Systems Control Lett. 14, 55-61. 